\title{
ANÁLISE ESTÁTICA DE VIGAS CONTÍNUAS CURVAS EM PLANTA COM SEÇÃO TRANSVERSAL ABERTA E PAREDE FINA
}

\author{
GILBERTO CARBONARI
}

\begin{abstract}
CARBONARI, G. Análise estática de vigas contínuas curvas em planta com seção transversal aberta e parede fina Semina v. 12, n. 4, p.189-204, dez. 1991.
\end{abstract}

\section{RESUMO}

Neste trabalho é apresentada uma solução numérica das equações diferenciais de vigas curvas assimétricas com parede fina e seção transversal aberta, devidas a Vlasov. É portanto admitido um comportamento estrutural elástico linear, sendo empregado na análise estática o método das Diferenças Finitas Centrais. Devido a curvatura da viga, a análise envolve o acoplamento entre a flexão vertical e a torção, levando em conta, além da torção de Saint Venant, também a torção de empenamento. São consideradas quaisquer combinações das condições de contorno e apoios intermediários, incluindo bordos rotulados e engastados na flexão e na torção. Os resultados da análise teórica, obtidos pela aplicação de um programa computacional implementado em microcomputador, são comparados com a bibliografia técnica, apresentando boa correlação.

PALAVRAS CHAVES: Vigas Curvas, Análise Estática, Seção Aberta, Parede Fina.

\section{1 -INTRODUÇÃO}

Este trabalho está baseado na Dissertação de Mestrado desenvolvida na UFRGS, indicada na referência CARBONARI ${ }^{1}$.

Verificou-se, na bibliografia técnica, trabalhos relacionados com a análise estática de vigas retas e curvas, com dupla simetria da seção transversal. No entanto, não foram encontradas soluções para seção transversal arbitrária, por exemplo, seção transversal aberta e de parede fina.

Tanto a curvatura da viga como a posição do centro de flexão (centro de torção) produzem acoplamento entre os esforços de flexão e torção, sendo então necessária a determinação do centro de flexão, resolvendo as forças internas apropriadas de flexão e de torção, e, finalmente, determina as tensões resultantes e deformações.

Adotou-se como solução da viga curva em planta com seção transversal aberta e de parede fina as equações diferenciais de VLASOV ${ }^{2}$, onde consideram 0 acoplamento entre a flexão e a torção.

Como solução dessas equações diferenciais, foi utilizado o método das Diferenças Finitas Centrais, admitindo-se contornos e apoios intermediários tanto rotulados como engastados na flexão e na torção.

Foi implementado um programa em microcomputador da família PC, designado VICURASS (Viga CURva
ASSimétrica), que faz a análise estática da viga e obtém as respostas em esforços internos solicitantes e deformações.

\section{2 -EQUAÇÕES DIFERENCIAIS DA VIGA CURVA ASSIMÉTRICA}

Considera-se viga curva circular de parede fina e seção aberta, com propriedades constantes ao longo da coordenada $s$ que define o comprimento de arco medido a partir do extremo da viga, como se ilustra na Fig. 2.1. Seja $R$ o raio de curvatura no plano normal ao eixo vertical Y. A teoria proposta por VLASOV $^{2}$ está baseada nas seguintes hipóteses:

- O material é elástico e linear;

- A espessura das paredes é pequena em relação às outras dimensões da seção transversal, sendo as dimensões da última pequenas em relação ao comprimento;

- A seção transversal é indeformável, isto é, é rígida no seu plano, mas pode empenar livremente;

- As deformações devidas às tensões de cisalhamento no plano médio da viga são desprezíveis.

Conforme as considerações acima, as equações diferenciais que governam o problema são as seguintes: 
$\mathrm{Z}\left(I_{z}+\frac{I_{w}}{R^{2}}\right) \frac{\partial^{4} y}{\partial a^{4}}-\frac{G R_{t}}{R^{2}} \frac{\partial^{2} y}{\partial s^{2}}+\frac{E I_{w}}{R} \frac{\partial^{4} \theta}{\partial z^{6}}-\frac{\left(E I_{z}+C R_{t}\right)}{R} \frac{\partial^{2} \theta}{\partial a^{2}}-P_{y}(e)$

$\mathrm{EI}_{\omega} \frac{\partial^{4} \theta}{\partial s^{4}}+\frac{E I_{\omega}}{R} \frac{\partial^{4} y}{\partial s^{4}}-\frac{\left(E I_{z}+G x_{c}\right)}{R} \frac{\partial^{2} y}{\partial s^{2}}-G_{t} \frac{\partial^{2} \theta}{\partial s^{2}}+\frac{\mathrm{EI}_{z}}{R^{2}} \theta=M_{t}$ (s)

onde,

$y(s)$ - deslocamento na direção vertical;

$\Theta(s)$ - giro na seção transversal ao redor do centro de flexão $A_{o j}$

$P_{y}(s)$ - projeção da carga externa distribuída na direção y;

$\mathrm{M}_{\mathrm{t}}(\mathrm{s})$ - momento torçor externo distribuído em torno de $\mathrm{A}_{0}$;

E - módulo de Young do material;

G - módulo de elasticidade transversal do material;

A - área da seção transversal;

R - Raio de curvatura;

$\mathrm{K}_{\mathrm{t}} \quad$ - constante de torção de St. Venant;

$\mathrm{I}_{\mathrm{z}} \quad$ - momento central de inérciaj

$I_{\omega} \omega$ - constante de empenamento (momento setorial de inércia);

sendo que as constantes geométricas $A, K_{t}, I_{z}$ e $I_{\omega}$ e o centro de flexão $A_{o}$ poderão ser obtidas segundo a referência (3).

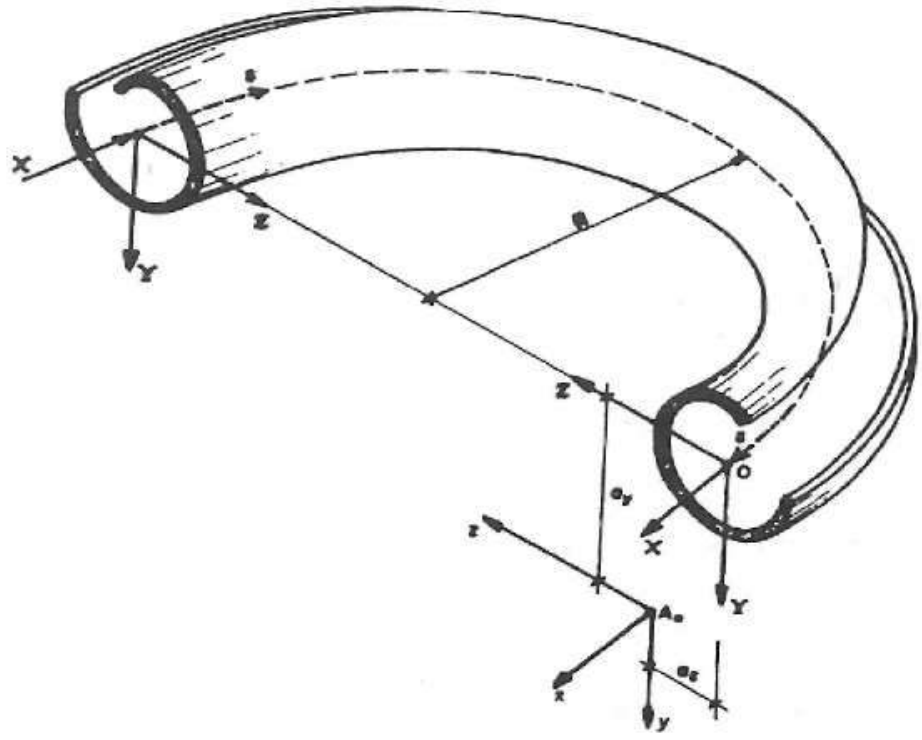

FIGURA 2.1 - Características geométricas e notação da viga curva de seção aberta e parede fina.

\section{3 - SOLUÇÃO NUMÉRICA DAS EQUAÇÕES DIFE- RENCIAIS DA VIGA CURVA}

Pelo acoplamento das equações diferenciais (2.1) e (2.2), a integração das mesmas por métodos analíticos diretos é, em geral, impraticável. Com isso, optou-se pela aplicação do método de Diferenças Finitas Centrais (D.F.C.). Neste caso, a mesma é feita na coordenada espacial s,.conforme retfcuia da FIGURA 3.1.

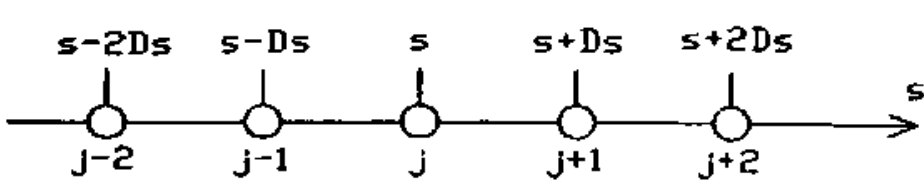

FIGURA 3.1 - Retfcula em Diferencas Finitas Centrais

Substituindo as operadores diferenciais nas equaçōes (2.1) e (2.2), para o ponto genérico j da retícula, chegase nas seguintes equaçōes em Diferenças Finitas Centrais, respectivamente.

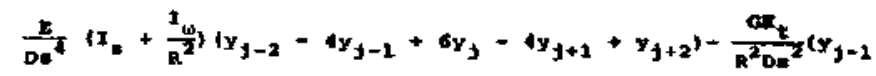

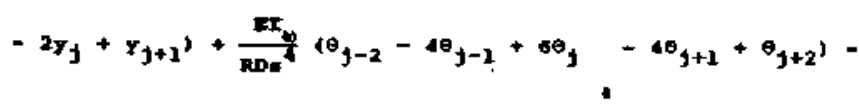

$$
\begin{aligned}
& -\frac{G I_{z}+G R_{t}}{\operatorname{gDQ}^{2}}\left(\theta_{j-1}-2 \theta_{j}+\theta_{j+1}\right)=P_{Y_{j}} \\
& \frac{B I_{w}}{D=4}\left(\theta_{j-2}-1 \theta_{j-1}+6 \theta_{j}-4 \theta_{j+1}+\theta_{j+2}\right)+\frac{B I_{w}}{R D D^{4}}\left(y_{j-2}=4 y_{j-1}+\right. \\
& \left.+6 y_{j}-4 y_{j+1}+y_{y+z}\right)-\frac{E I_{z}+G K_{t}}{R D z^{2}}\left(y_{j-1}-2 Y_{j}+y_{j+1}\right)- \\
& -\frac{G R_{t}}{D R^{2}}\left(\theta_{j-1}-2 \theta_{j}+\theta_{j+1}\right)+\frac{B I_{z}}{R^{2}} \theta_{j}-M_{t_{j}}
\end{aligned}
$$

Reagrupando os termos das equações (3.1) e (3.2), na forma matricial, para o no genérico j da retícula, chegase no seguinte sistema local:

$$
\mathrm{K} \quad \overrightarrow{\mathrm{U}}=\overrightarrow{\mathbf{P}} \text {, }
$$

onde o vetor $\overrightarrow{\mathrm{U}}$ contém as incógnitas nodais em deslocamentos e giros, cujos elementos possuem a seguinte disposição:

$$
\overrightarrow{\mathbf{u}}=\left\{\begin{array}{c}
y_{j-2} \\
\theta_{j-2} \\
- \\
y_{j-1} \\
\theta_{j-1} \\
-y_{j} \\
\theta_{j} \\
---- \\
y_{j+1} \\
\theta_{j+1} \\
---- \\
y_{j+2} \\
\theta_{j+2}
\end{array}\right\}
$$


A matriz $\mathrm{K}$ corresponde à matriz de rigidez local, cujas componentes estão definidas da seguinte forma:

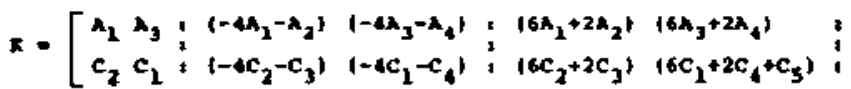

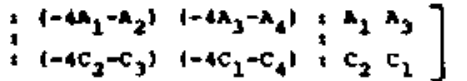

(s)

Once:

$A_{2}=\frac{I}{D R^{d}}\left(I_{2}+\frac{I_{G}}{R^{2}}, A_{2}=\frac{G R_{t}}{D R^{2} R^{2}}, A_{3}=\frac{R I}{R D}\right.$,

$\lambda_{1}=\frac{1}{\operatorname{son}^{2}}\left(\mathrm{Ir} \mathbf{x}_{2}+G R_{2}\right)$

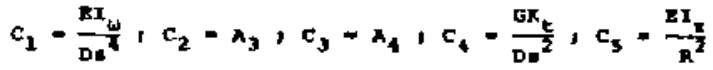

E, por final, resta o vetor $\vec{P}$, que contém as componentes nodais das cargas externas a momentos torçores externos distriburdos por unidade de comprimento da viga, relativos ao nó genérico j, possuindo a seguinte defínição:

$$
\overrightarrow{\mathbf{P}}=\left\{\begin{array}{l}
\mathbf{p}_{j} \\
\mathrm{Mt}_{j}
\end{array}\right\}
$$

Quando a viga é discretizada em Diferenças Finitas Centrais com $N$ nós, isto $\hat{e}$, fazendo o nó genérico j variar de 1 ate $N$, conforme FIGURA 3.2.
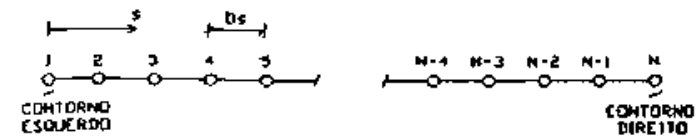

FIGURA 3,2 - Discretização espacial da viga curva em D.F.C. então o sistema de soluçāo (3.3) assume a seguinte configuração global;

$$
\boldsymbol{K}^{\star} \overline{\mathbf{y}}^{*}=\mathbf{B}^{*}
$$

onde $K^{*}$ equivale à matriz de rigidez global da viga curva, cuja montagem obedece ao seguinte procedimento:

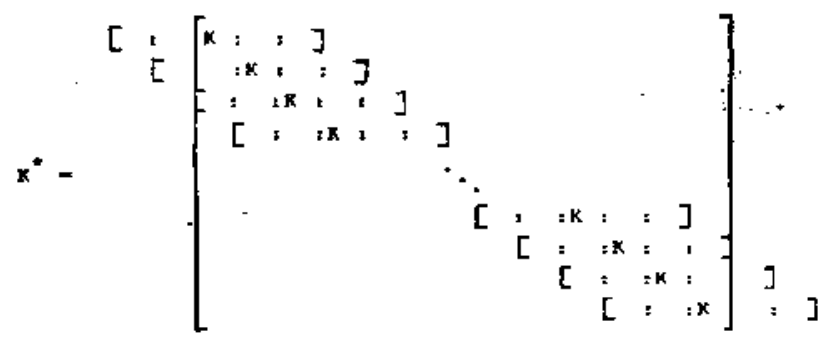

FIGURA 3.3 - Matriz global onde a dimensão da matriz $\mathrm{K}^{*}$ é de $2(\mathrm{~N}-2) \times 2(\mathrm{~N}-2)$.

Já os vetores $\vec{U}^{*}$ e $\vec{P}$, considerando nulos os deslozamentos verticais e giros de torção nos extremos da vija, possuem a seguinte montagem global:

$$
\overrightarrow{\mathrm{U}}^{*}=\left\{\begin{array}{c}
\mathrm{y}_{2} \\
\theta_{2} \\
\mathrm{y}_{3} \\
\theta_{3} \\
\vdots \\
\vdots \\
\mathrm{Y}_{\mathrm{N}-2} \\
\theta_{\mathrm{N}-2} \\
\mathrm{Y}_{\mathrm{N}-1} \\
\theta_{\mathrm{N}-1}
\end{array}\right\} \overrightarrow{\mathrm{P}}^{*}=\left\{\begin{array}{c}
\mathrm{P}_{2} \\
\mathrm{Mt}_{2} \\
\mathrm{PY}_{3} \\
\mathrm{Mt}_{3} \\
\vdots \\
\vdots \\
\mathrm{PY}_{\mathrm{N}-2} \\
\mathrm{Mt}_{\mathrm{N}-2} \\
\mathrm{PY}_{\mathrm{N}-1} \\
\mathrm{Mt} \\
\mathrm{N}-1
\end{array}\right\}
$$

onde os elementos do vetor $\vec{U}^{*}$ são as componentes nodais dos deslocamentos e giros com $\mathrm{N}$ nós discretizados; e os elementos de $\overrightarrow{\mathrm{P}}^{*}$ são as componentes nodais das cargas e momentos torçores distribuifos por unidades de comprimento da viga curva. Tanto o vetor $\overrightarrow{\mathrm{U}}^{*}$ como o vetor $\overrightarrow{\mathrm{P}}^{*}$ possuem dimensão de $2(\mathrm{~N}-2)$ elementos.

No caso de cargas concentradas aplicadas na viga, para obter as componentes das cargas nodais do vetor $\overrightarrow{\mathrm{P}}$, se determinam as componentes nodais equivalentes através da posição da carga, tornando-as distribuifdas.

Finalmente, para que o sistema global (3.7) seja resolvido, resta a consideração das condições de contorno.

\section{Contorno rotulado:}

$$
U_{j}=0 \text { e } U_{j+1}=\cdot-U_{j-1}
$$

Contorno engastado:

$$
\mathrm{U}_{j}=0 \text { e } \mathrm{U}_{j+1}=\mathrm{U}_{j-1}
$$

onde $U$ é um deslocamento genérico que representa as incógnitas y e $\Theta$.

Para os casos onde há apoios intermediários, considera-se para o nó $\mathrm{j}: \mathrm{k}_{\mathrm{j}}=1$ e $\mathrm{P}_{\mathrm{j}}=0$, ou seja, $\mathrm{U}_{\mathrm{j}}=0$.

Devido a matriz de rigidez global ser simétrica, o sistema matricial (3.7) foi resolvido pelo método de $\mathrm{CHO}$ LESKY, fazendo inicialmente a triangularização e, após, a retrosubstituição para diferentes estados de carregamento.

Após calculados os deslocamentos verticais e os giros de torção, torna-se possível a obtenção dos esforços internos solicitantes (Cortante, Momento Fletor, Momento Torçor de St. Venant, Momento Torçor de Empenamento, Momento Torçor Total e o Bimomento), cujas expressões em Diferenças Finitas Centrais, relativo ao nó genérico j, ficam representadas da seguinte forma. 


\section{(cortante)}

$Q_{Y}=-E_{Z}\left(\frac{\partial^{3} Y}{\partial s^{3}}-\frac{1}{R} \frac{\partial \theta}{\partial s}\right)+\frac{T}{R}$,

(momento fletor)

$\mathbf{M}_{z}=-\mathbf{E I}_{\mathbf{z}}\left(\frac{\partial^{2} y}{\partial s^{2}}-\frac{\theta}{R}\right)$

(torção de St. Venant)

$T_{s}=G K_{t}\left(\frac{\partial \theta}{\partial s}+\frac{1}{R} \frac{\partial Y}{\partial s}\right)$.

\section{(torçăo de empenamento)}

$\mathbf{T}_{\theta}=-\mathrm{E} I_{\omega}\left(\frac{\partial^{3} \theta}{\partial s^{3}}+\frac{1}{R} \frac{\partial^{3} y}{\partial s^{3}}\right)$

\section{(torghăo total)}

$\mathbf{T}=\mathbf{T}_{\mathrm{S}}+\mathbf{T}_{\theta}$.

\section{(biomomento)}

$\mathrm{B}_{\mathrm{M}}={ }^{-}-\mathrm{EI}_{\omega}\left(\frac{\partial^{2} \theta}{\partial s^{2}}+\frac{1}{R} \frac{\partial^{2} y}{\partial s^{2}}\right)$

$Q_{Y_{j}}=-E I_{z}\left[\frac{1}{2 D s^{3}}\left(y_{j+z}-2 y_{j+1}+2 y_{j-1}-y_{j-2}\right)-\right.$

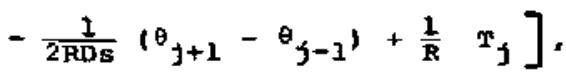

$M_{z_{j}} \approx-E I_{z}\left[\frac{1}{0 s^{2}}\left(y_{j-1}-2 y_{j}+y_{j+1}\right)-\frac{1}{R} \theta_{j}\right]$.

$\mathbf{T}_{a_{j}}=G K_{t}\left[\frac{1}{2 D s}\left(\theta_{j+1}-\theta_{j-1}\right)+\frac{1}{2 D A R}\left(y_{j+1}-y_{j-1}\right)\right]$.

$r_{\theta_{j}}=-E_{4}\left[\frac{1}{2 D s^{3}}\left(\theta_{j+2}-2 \theta_{j+1}+2 \theta_{j-1}-\theta_{j-2}\right)+\right.$

$\left.+\frac{3}{2 \mathrm{RDs}}{ }^{3}\left(y_{j+2}-2 y_{j+1}+2 y_{j-1}-y_{j-2}\right)\right]$.

$\mathbf{T}_{j}=\mathbf{T}_{\mathbf{s}_{j}}+\mathbf{T}_{\mathbf{\theta}_{j}}$

$\mathrm{B}_{j}=-\mathrm{EI}_{\mathrm{w}}\left[\frac{1}{\mathrm{Ds}}\left(\theta_{j-1}-2 \theta_{j}+\theta_{j+1}\right)+\frac{1}{\mathrm{RDs}^{2}}\left(y_{j-1}-\right.\right.$

$\left.2 y_{j}-y_{j+1} j\right]$.
Com isso, a solução estática da viga curvada em planta se completa, e a partir dela foi implementado em programa em microcomputador designado de VICURASS, cujos exemplos resolvidos estão mostrados na seção que segue (4.).

\section{4 - EXEMPLOS COMPARATIVOS}

4.1 - Exemplo 1

Dada a viga circular fechada de raio $5 \mathrm{~m}$, simplesmente apoiada nos pilares A, B e C, conforme Fig. 4.1.1, com seção transversal retangular, cuja rigidez a flexão é igual a rigidez a torção, isto é, $E I_{z} / G_{t}=1$, é submetida a uma carga uniformemente distribuída vertical de $14.4 \mathrm{KN} / \mathrm{m}$ e a um momento distribuído tangente a viga de 0.24 $\mathrm{KNm} / \mathrm{m}$.

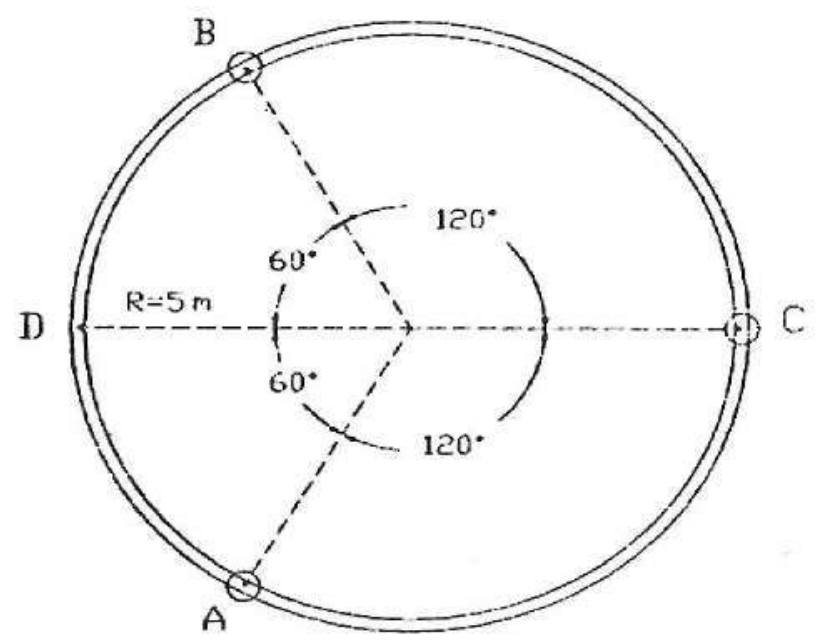

FIGURA 4.1.1 - Viga curva fechada com 3 apoios

Os resultados obtidos pelo presente trabalho, Figs. 4.1.2 a 4.1.4, através do programa VICURASS, são comparados com HIRSCHFELD ${ }^{4}$, conforme a Tabela 4.1.

TABELA 4.1 - Momentos fletores e Torçores (KNm)

\begin{tabular}{|c|c|c|c|}
\hline & I MXRSCGIFELD" & victeress & D15. (1) \\
\hline 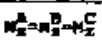 & -143.55 & -145.54 & 0.01 \\
\hline$\omega_{2}^{0}$ & 74.10 & $34+12$ & 0.03 \\
\hline$n_{t}^{n-5}$ & 29.79 & 29.28 & 0.30 \\
\hline
\end{tabular}




\section{2 - Exemplo 2}

Dada a mesma viga do exemplo 1 simplesmente apoiada em 6 pilares, conforme Fig. 4.2.1, submetida a uma carga uniformemente distribuída vertical de $14.4 \mathrm{KN} / \mathrm{m}$.

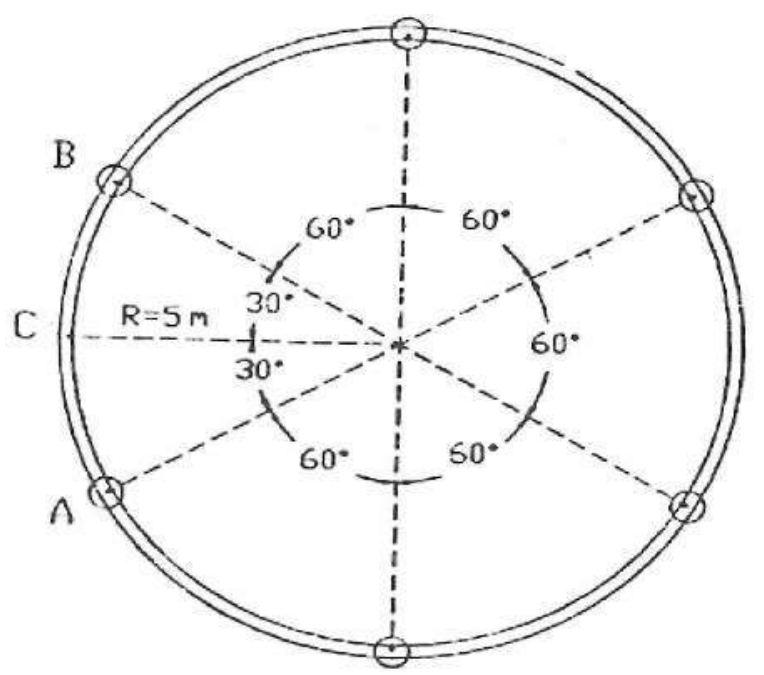

FIGURA 4.2.1 - Viga curva fechada com 6 apoios

Os resultados obtidos pelo presente trabalho, Figs. 4.2.2 a 4.2.4, são comparados com COURTAND \& LEBELLE $^{5}$, conforme a Tabela 4.2.

TABELA 4.2 - Momentos fletores e Torçores (KNm)

\begin{tabular}{|c|c|c|c|}
\hline & COURTAND a Lraghiz ${ }^{5}$ & recuruss & Dif. (v) \\
\hline & -33.522 & -33.514 & 0.02 \\
\hline$x_{x}^{c}$ & 16.927 & 16.995 & 0.05 \\
\hline$t_{t}^{n-1}$ & 3.416 & 3.413 & 0.09 \\
\hline
\end{tabular}

\section{3 - Exemplo 3}

Dada a mesma viga do Exemplo 1 engastada no pilar A, conforme Fig. 4.3.1, submetida a uma carga uniformemente distribuída vertical de $10 \mathrm{Kn} / \mathrm{m}$.

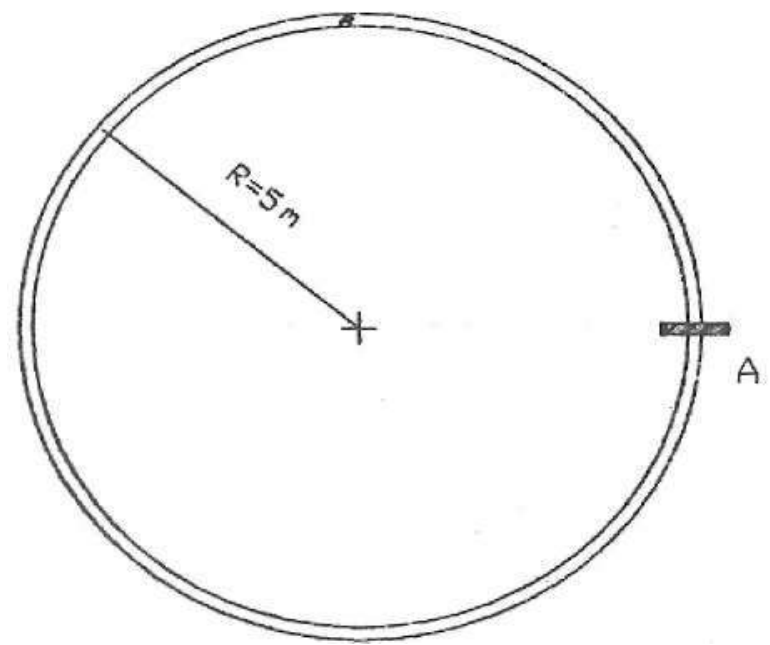

FIGURA 4.3.1 - Viga curva fechada engastada no pilar A Semina, Ci. Exatas/Tecnol., v.12, n.4, p.189-204, dez. 1991
Os resultados obtidos pelo presente trabalho, Figs. 4.3.2 a 4.3.7, são comparados com COURTAND \& LEBELLE ${ }^{5}$, conforme a Tabela 4.3.

TABELA 4.3 - Momentos fletores e Torçores (KNm)

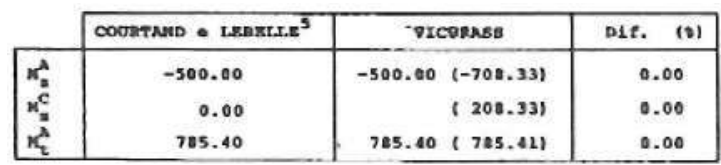

Obs.: Os valores entre parênteses correspondem a $E 1_{z}$ / GKj $=11$, Figs. 4.3 .5 a $4.3,7$, enquanto que para os demais valores, $\mathrm{EI}_{\mathrm{z}} / \mathrm{GK}_{\mathrm{t}}=1$, Figs. 4.3.2 a 4.3.4.

4.4 - Exemplo 4

Dada a mesma viga do Exemplo 1, conforme Fig. 4.4.1, submetida a uma carga concentrada de $30 \mathrm{KN}$, aplicada no ponto $P$.

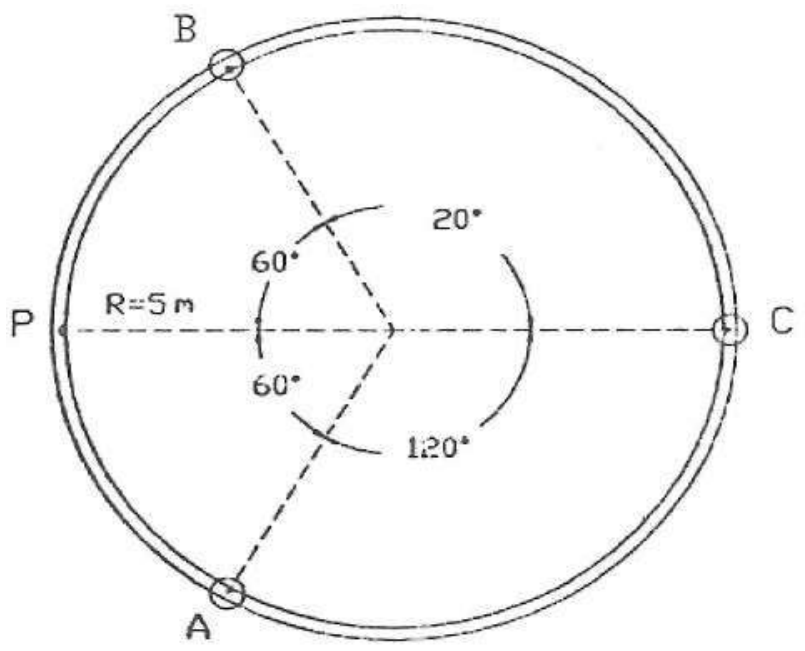

FIGURA 4.4.1 — Viga curva fechada com 3 apoios

Os resultados obtidos pelo presente trabalho, Figs. 4.4.2 a 4.4.4, são comparados com HIRSCHFELD ${ }^{4}$, conforme a Tabela D.§.

TABELA 4.4 Momentos fletores e torçores (KNm)

\begin{tabular}{|c|c|c|c|}
\hline & 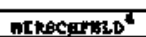 & vecososts & Dte, 10 \\
\hline & 26.90 & 28.06 & 0.14 \\
\hline & -26.10 & -36.10 & 0.00 \\
\hline $\mathbf{u}_{\mathbf{z}}^{\mathbf{p}}$ & 57.70 & 57.73 & 0.05 \\
\hline$x_{t}^{-a x}$ & 29.63 & 19.65 & .10 \\
\hline
\end{tabular}




\section{Rroggana VICuRAss - UIga Cyllya Assimetrica, Autor: Gilberto Cambonari \\ CARREGAHE H I DA UIG - 679 nos discretizados}

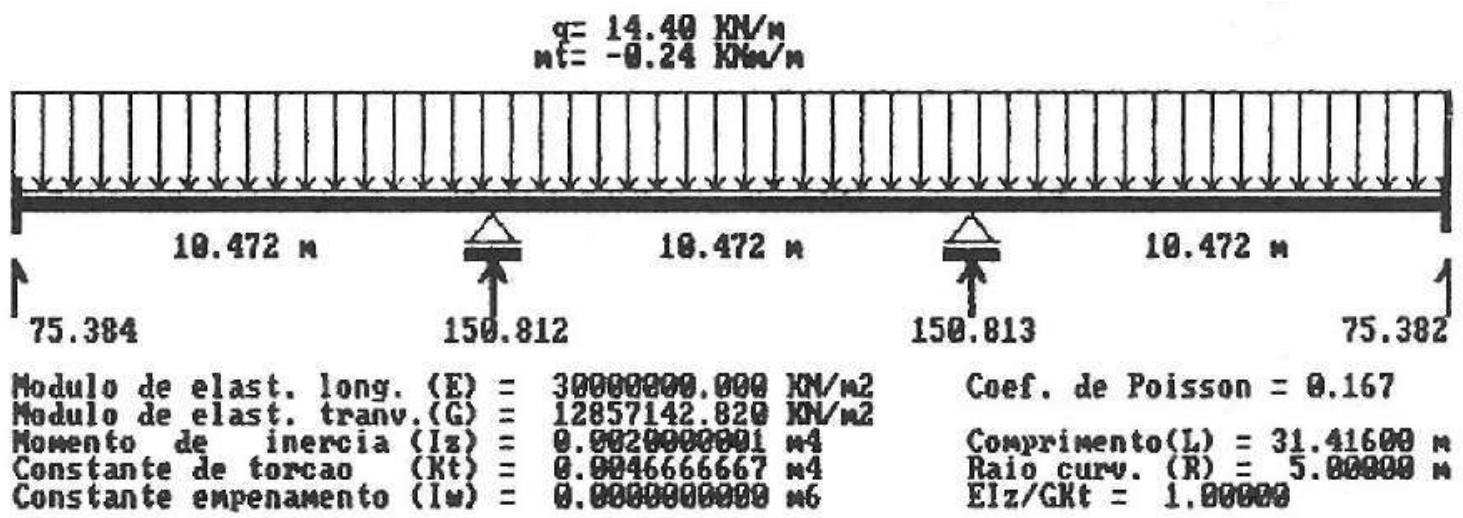

FIGURA 4.1.2 - Dados da viga (EXEMPLO 1)

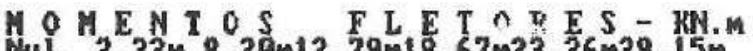

Hul. $2.32 \mathrm{~m} 8.20 \mathrm{~m} 12.79 \mathrm{m18} .67 \mathrm{ma3} .26 \mathrm{~m} 29.15 \mathrm{~m}$

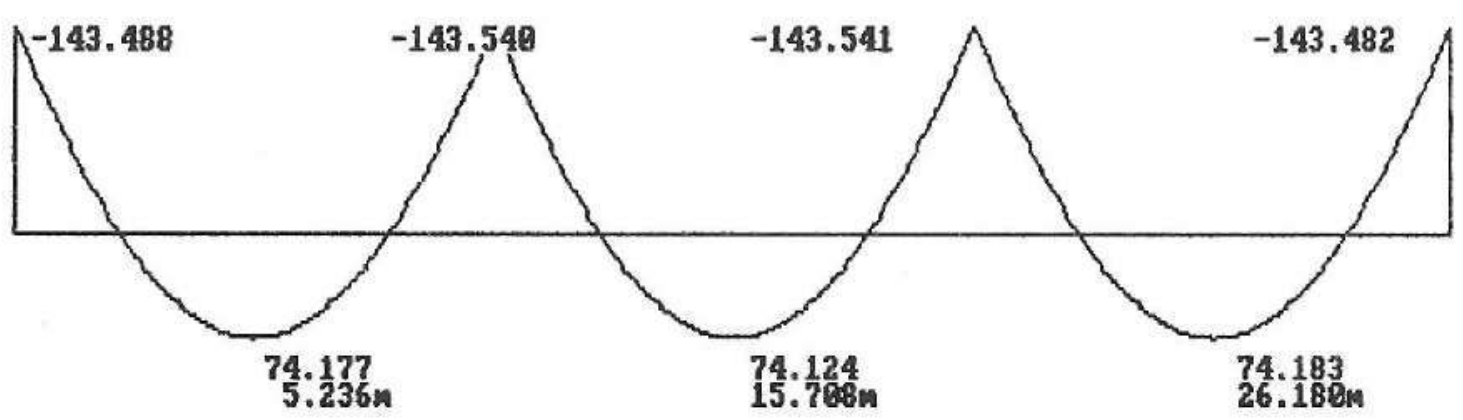

FIGURA 4.1.3 — Diagrama de momentos fletores

I O RCAO SI, VENAN T -KN.

Max=29.8863kin Pos=29.1455m

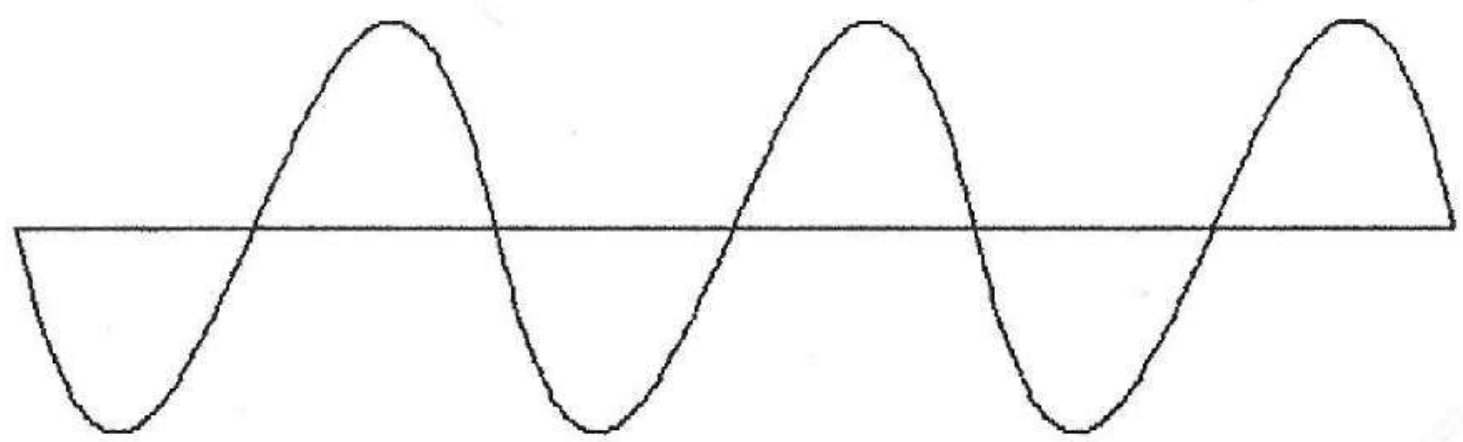

FIGURA 4.1.4 - Diagrama de momentos torçores

Semina, Ci. Exatas/Tecnol., v.12, n.4, p.189-204, dez. 1991 
Prograna VICURASS - UIga CuRYa ASSimetrica, Autor: Gilberto Carbonari C A R REGHE I T DA OIGA - 679 nos discretizados

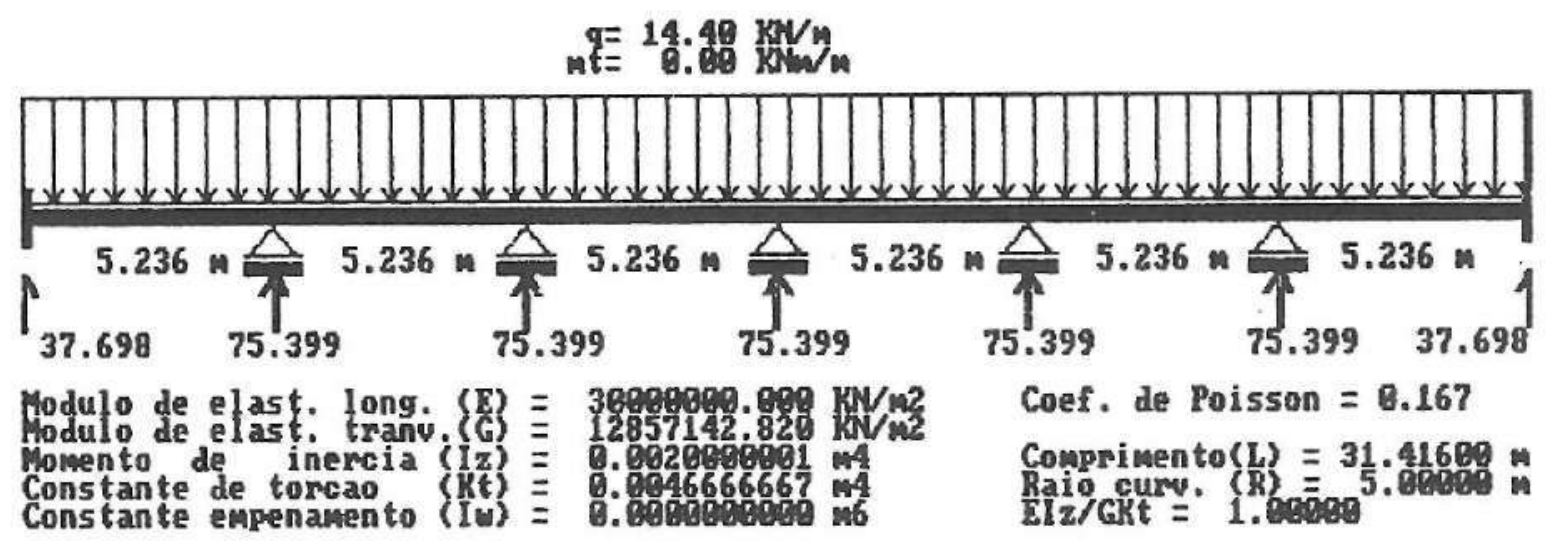

FIGURA 4.2.2 - Dados da viga (EXEMPLO 2)

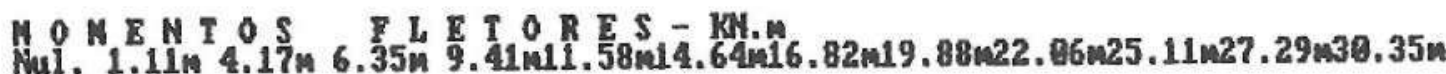

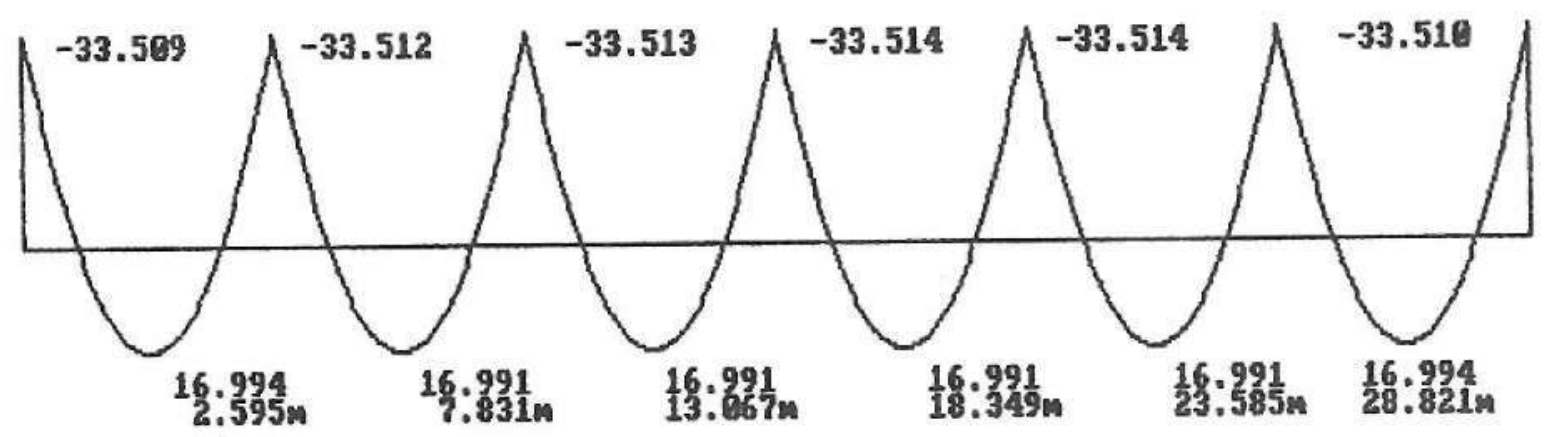

FIGURA 4.2.3 - Diagrama de momentos fletores

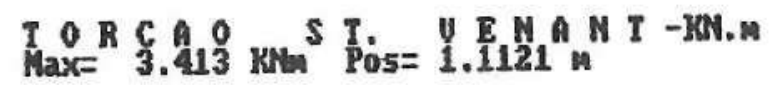

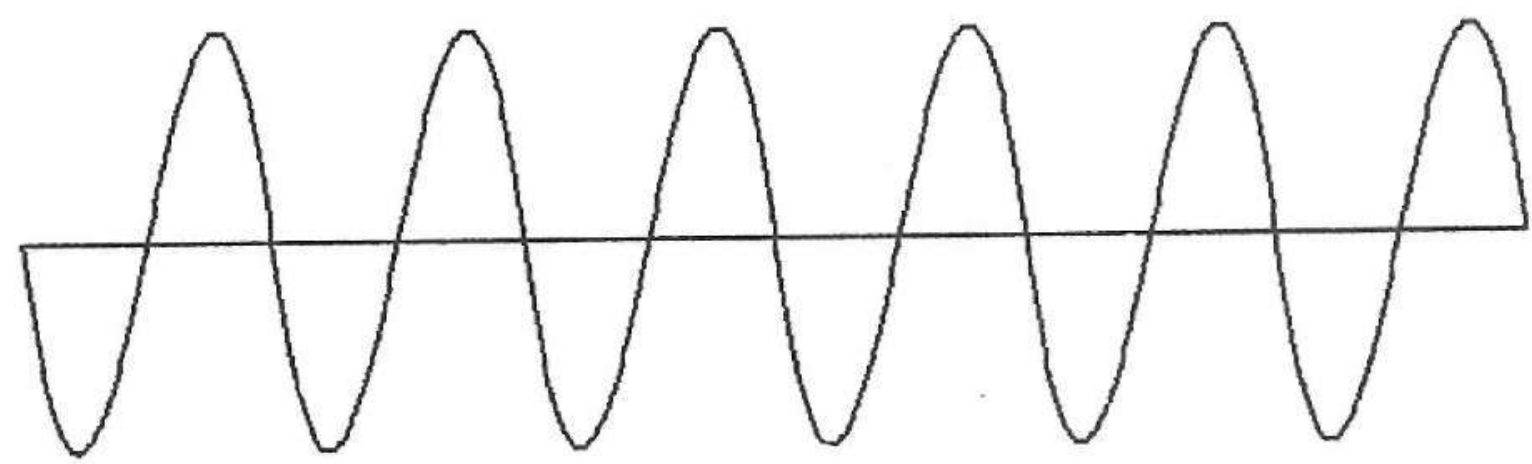

FIGURA 4.2.4 - Diagrama de momentos torçores 
Programa UICURASS - UIga CURya Assimetrica, Autor: Gilberto Carbonari

CARREGAHENT8 DA UIGA- 679 nos discretizados

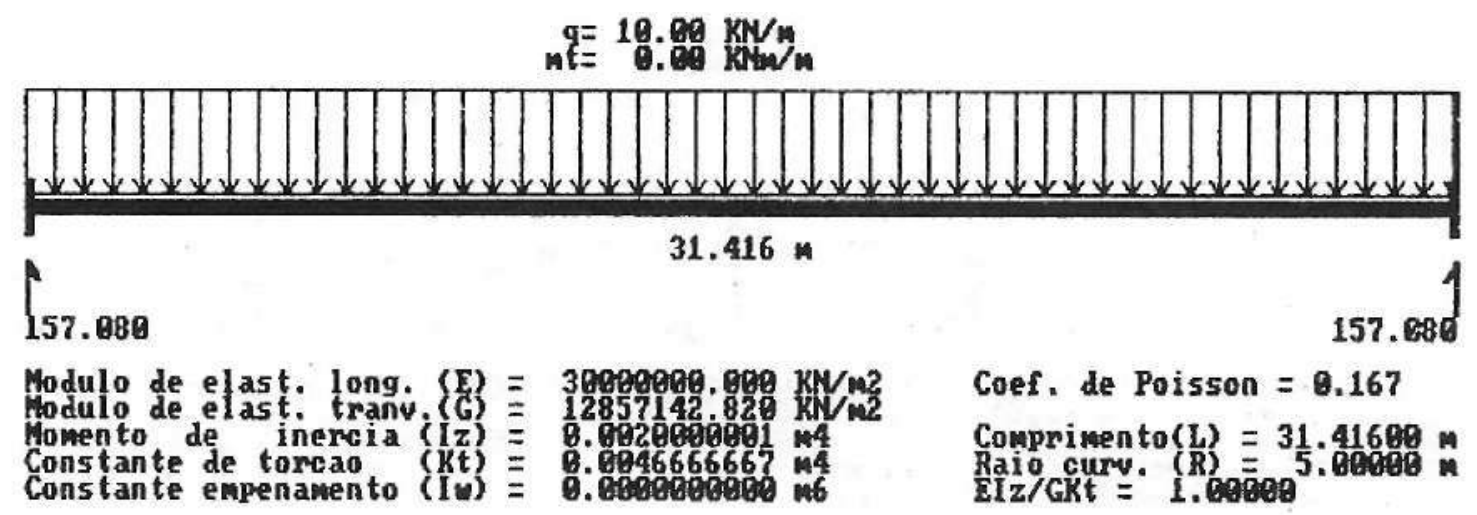

FIGURA 4.3.2 Dados da viga (EXEMPLO 3)

\section{9.}

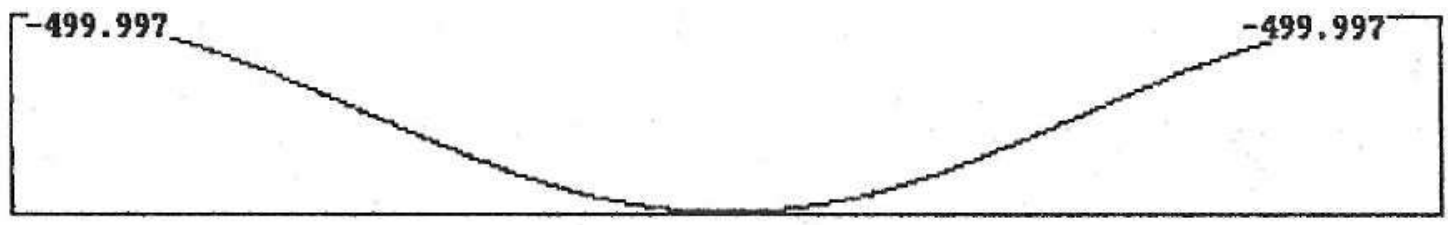

FIGURA 4.3.3 - Diagrama de momentos fletores

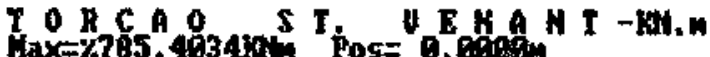

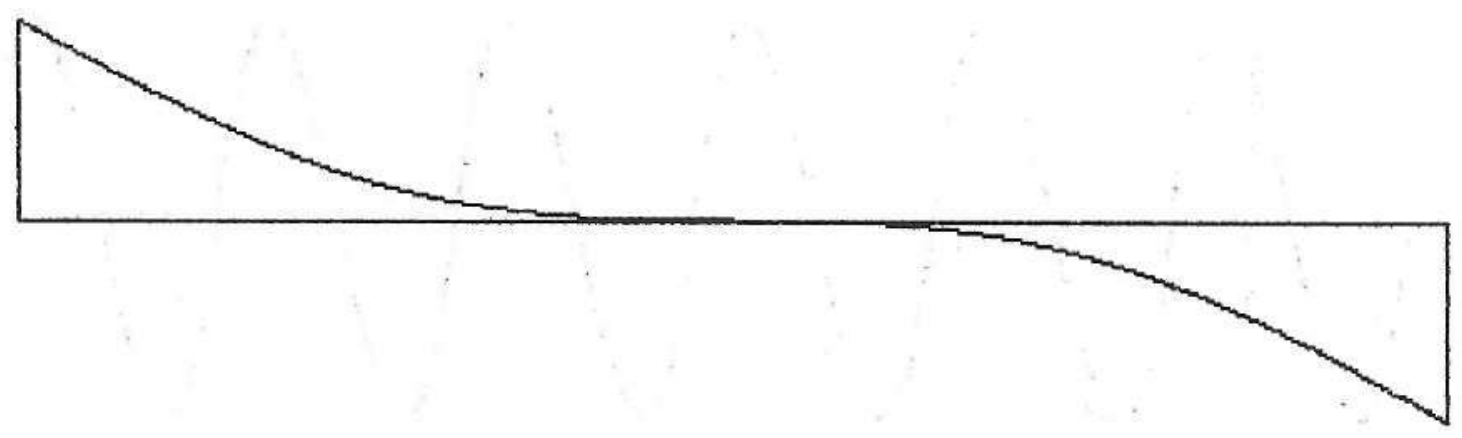

FIGURA 4.3.4 - Diagrama de momentos torçores 
Prograna UICURaSS - UIga CuRya ASSimetrica, Autor: Gilberto Carbonari

CRRAEGAHEN TO DA U I G - 679 nos discretizados

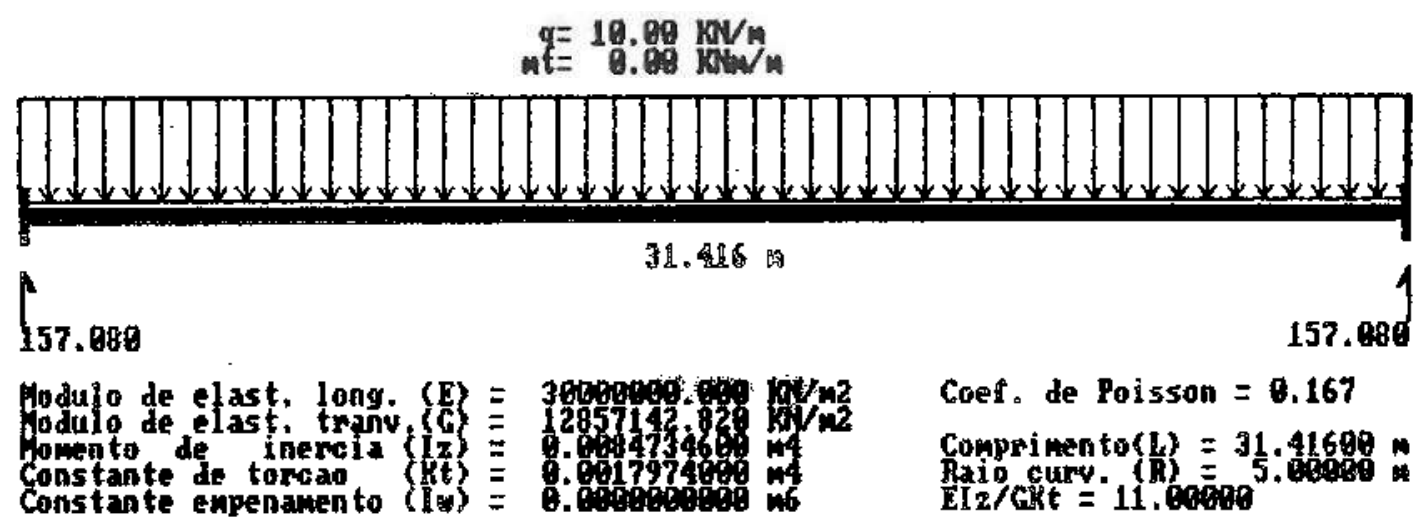

FIGURA 4.3.5 - Dados da viga (EXEMPLO 3)

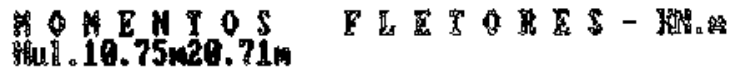

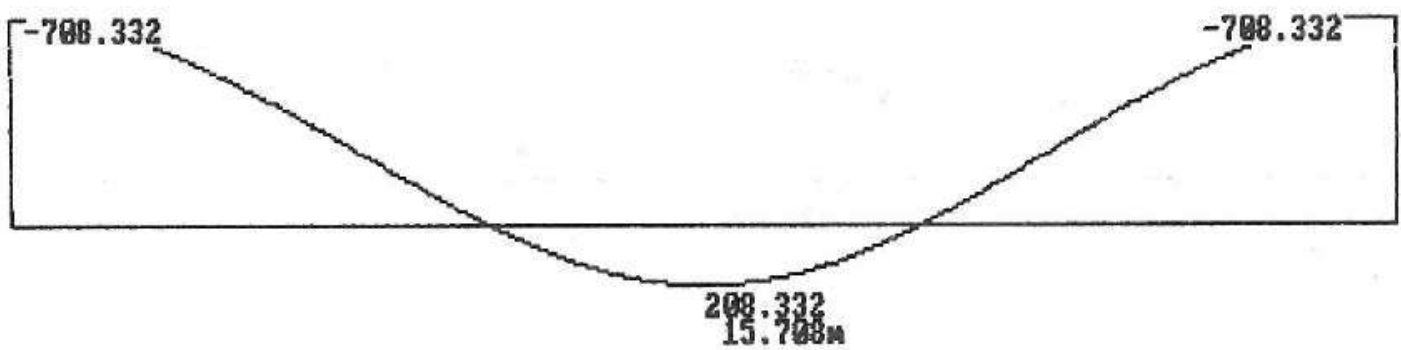

FIGURA 4.3.6 Diagrama de momentos fletores

T O RCAO S I. U ENAN T - KM.H

hasc=785. $406 \mathrm{KN}$ Pos=0.0000 $\mathrm{N}$

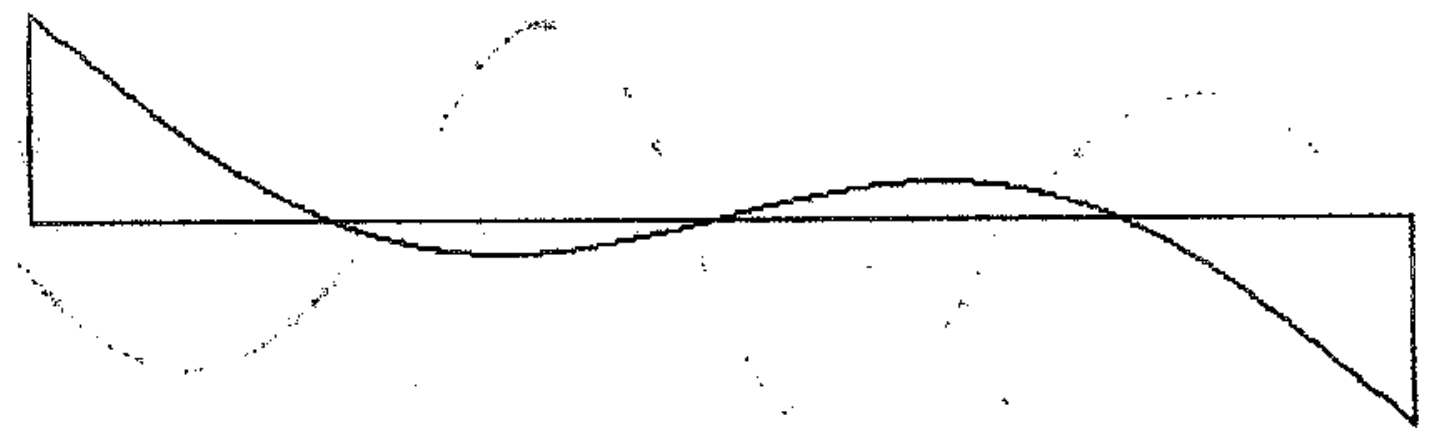

FIGURA 4.3.7 — Diagrama de momentos torçores 


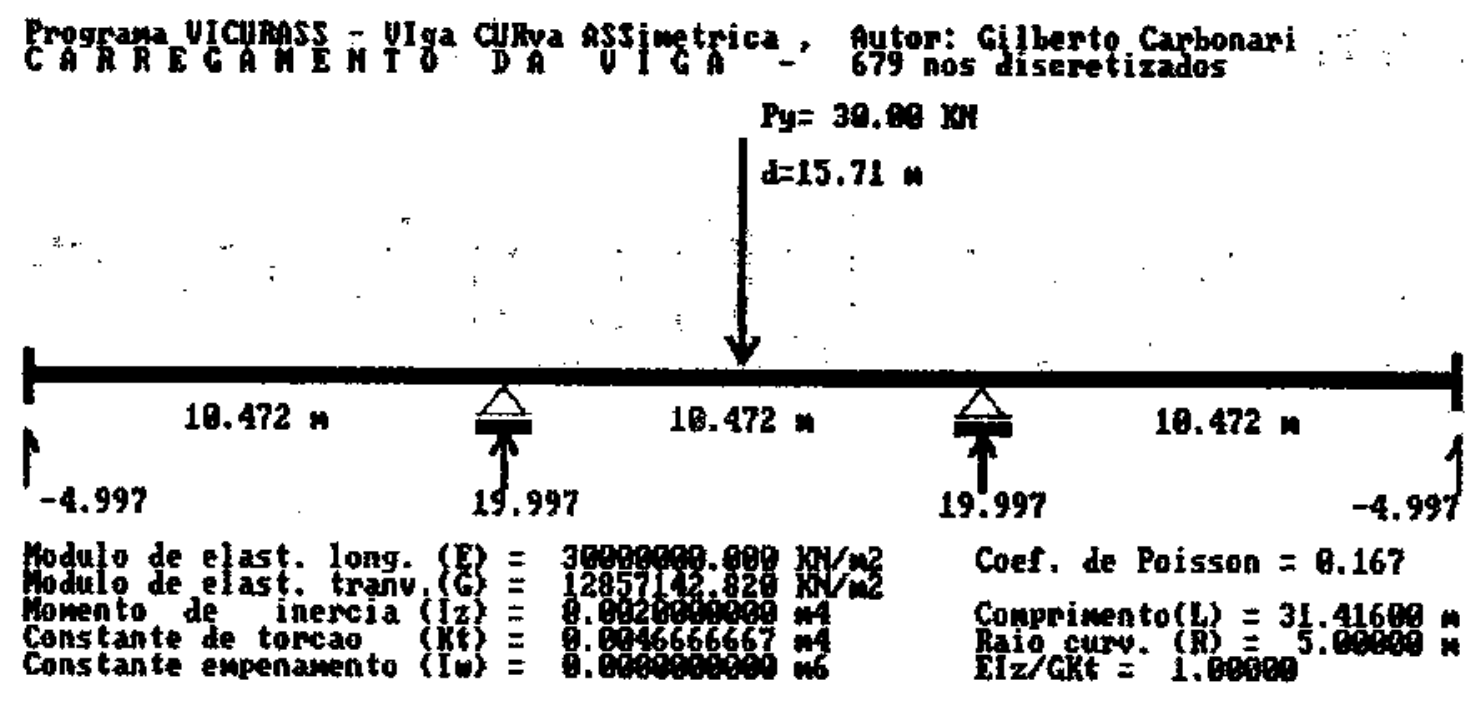

FIGURA 4.4.2 - Dados da viga (EXEMPLO 4)

10.

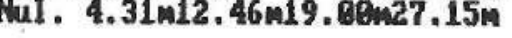

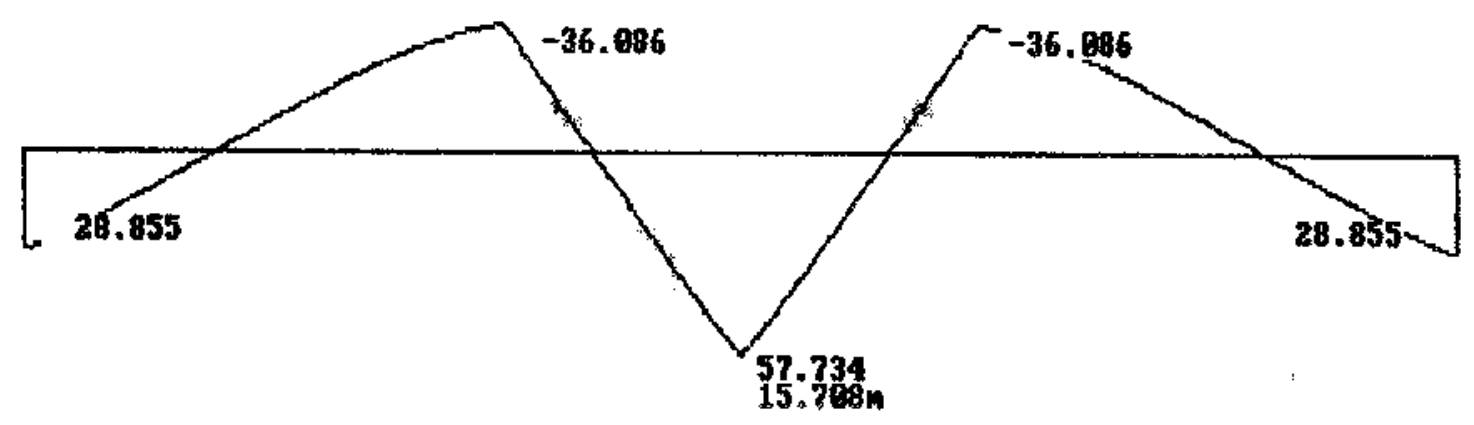

FIGURA 4.4.3 — Diagrama dos momentos fletores

IORCAO ST. U E A A T - KN4

Max= 19.648 Jin Pos=12.4181

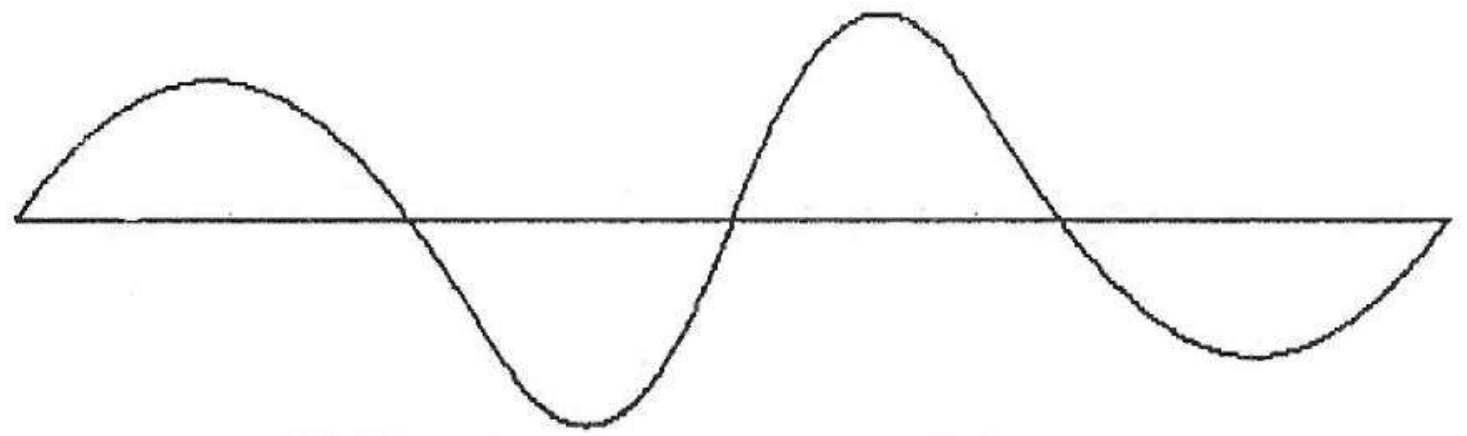

FIGURA 4.4.4 — Diagrama dos momentos torçores 
4.5 - Exemplo 5: Torção não Uniforme (com empenamento)

Dada a viga curva da Fig. 4.5.1 com 20m de comprimento e raio de curvatura de $22.9183 \mathrm{~m}$, referente a um ângulo de abertura de $50^{\circ}$, e com as seguintes propriedades físicas e geométricas: $E=2.1 \times 10^{8} \mathrm{KN} / \mathrm{m}^{2}, V=0,3, \mathrm{G}=$ $8.077 \times 10^{7} \mathrm{KN} / \mathrm{m}^{2}, \mathrm{I}_{\mathrm{z}}=10^{-4} \mathrm{~m}^{4}, \mathrm{~K}_{\mathrm{t}}=1.96 \times 10-{ }^{5} \mathrm{~m}^{4}$, $I_{W}=1.6 \times 10^{-6} \mathrm{~m}^{6}$. A viga está sumetida a uma carga uniformemente distribuída de $15 \mathrm{KN} / \mathrm{m}$ com excentricidade $6.66 \mathrm{~cm}$ em relação ao centro de torção e a uma carga concentrada de $100 \mathrm{KN}$ distante $3 \mathrm{~m}$ do apoio B com excentricidade $5 \mathrm{~cm}$ em relação ao centro de torção.

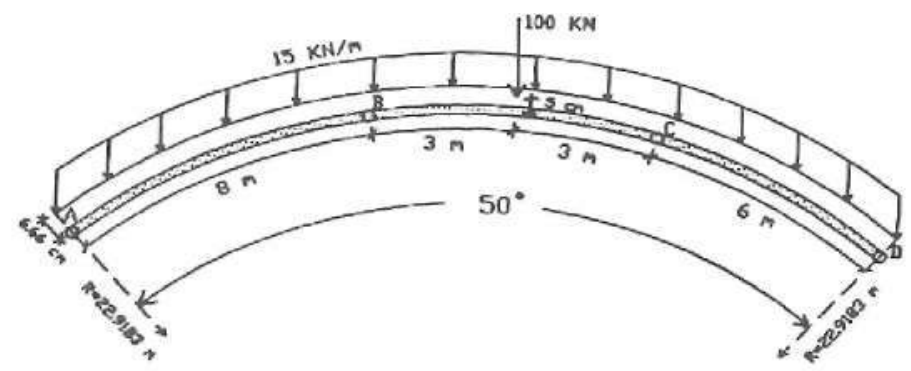

FIGURA 4.5.1 - Viga curva aberta com 4 apoios

Foram analisados 3 casos diferentes, através do programa computacional VICURASS, sendo que os resultados obtidos estão mostrados nas 'Figs. 4.5.2 a 4.5.4. Nos 3 casos há restrição ao deslocamento vertical $(y=0)$ e ao giro por torção $(\Theta=0)$ nos apoios $A, B, C$ e D.

CASO 1. VIGA CURVA $\left(\alpha=50^{\circ}\right)-$ Fig. 4.5 .2 Apoios A e D. Livre para empenar

CASO 2: VIGA CURVA $\left(\alpha=50^{\circ}\right)-$ Fig. 4.5.3 Apoios A e D: Restrição ao empenamento

CASO 3: VIGA RETA $(\mathrm{R}=\infty)$ - Fig. 4.5.4 Apoios A e D: Livre para empenar

\section{5 - TABELAS PRÁTICAS PARA OS PROFISSIONAIS}

5.1 - Viga Circular de Raio R Simplesmente Apoiada em n Pilares Equidistantes, Fig. 5.1, Submetida a uma Carga Uniformemente Distribuída q.

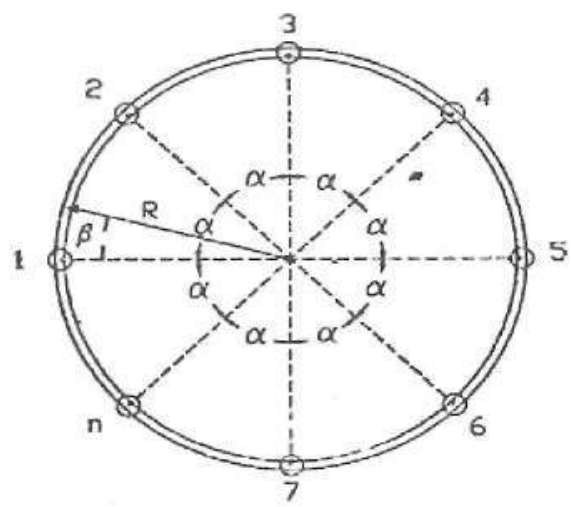

FIGURA 5.1 - Viga Circular Fechada

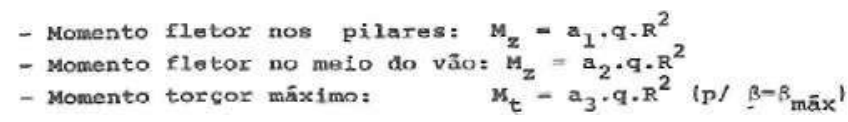

Semina, Ci. Exatas/Tecnol., v.12, n.4, p.189-204, dez. 1991
TABELA 5.1 - Coeficientes $a_{1}, a_{2}$ e $a_{3}$ função do valor de $n$

\begin{tabular}{|c|lllll|}
\hline $\mathrm{n}$ & \multicolumn{1}{c}{$\mathrm{a}$} & ${ }_{1}$ & $a_{2}$ & $\beta_{\operatorname{mix}}$ & $\mathrm{a}_{3}$ \\
\hline 2 & $180^{\circ}$ & -1.0000 & 0.57080 & $50.46^{\circ}$ & 0.330674 \\
3 & $120^{\circ}$ & -0.3954 & 0.20920 & $34.21^{\circ}$ & 0.082768 \\
4 & $90^{\circ}$ & -0.2146 & 0.11072 & $25.80^{\circ}$ & 0.033125 \\
5 & $72^{\circ}$ & -0.1352 & 0.06896 & $20.69^{\circ}$ & 0.016567 \\
6 & $60^{\circ}$ & -0.0931 & 0.04720 & $22.27^{\circ}$ & 0.009468 \\
7 & $51.43^{\circ}$ & -0.0681 & 0.03438 & $14.81^{\circ}$ & 0.005918 \\
8 & $15^{\circ}$ & -0.0519 & 0.02617 & $12.97^{\circ}$ & 0.003946 \\
9 & $40^{\circ}$ & -0.0409 & 0.02060 & $11.53^{\circ}$ & 0.002762 \\
10 & $36^{\circ}$ & -0.0331 & 0.01664 & $10.38^{\circ}$ & 0.002009 \\
11 & $32.73^{\circ}$ & -0.0273 & 0.02372 & $9.44^{\circ}$ & 0.001507 \\
12 & $30^{\circ}$ & -0.0230 & 0.01152 & $8.65^{\circ}$ & 0.001159 \\
13 & $27.69^{\circ}$ & -0.0195 & 0.00980 & $7.99^{\circ}$ & 0.000911 \\
14 & $25.71^{\circ}$ & -0.0168 & 0.00844 & $7.42^{\circ}$ & 0.000729 \\
15 & $24^{\circ}$ & -0.0147 & 0.00735 & $6.92^{\circ}$ & 0.000592 \\
16 & $22.5^{\circ}$ & -0.0129 & 0.00645 & $6.49^{\circ}$ & 0.000487 \\
17 & $21.18^{\circ}$ & -0.0114 & 0.00571 & $6.11^{\circ}$ & 0.000405 \\
18 & $20^{\circ}$ & -0.0102 & 0.00510 & $5.77^{\circ}$ & 0.000342 \\
19 & $18.95^{\circ}$ & -0.0091 & 0.00457 & $5.47^{\circ}$ & 0.000291 \\
20 & $18^{\circ}$ & -0.0082 & 0.00412 & $5.19^{\circ}$ & 0.000249 \\
\hline
\end{tabular}

5.2 - Viga Circular de Raio R Bi-engastada a Flexão e a Torção, Fig. 5.2, Submetida a uma Carga Uniformemente Distribuída q.

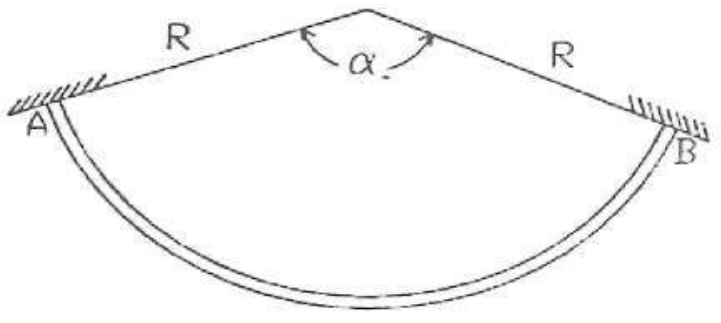

FIGURA 5.2 - Viga Circular Bi-engastada (Tab. 5.2)

\subsection{1 — Exemplo de Aplicação da Tab. 5.2}

Seja uma viga curva bi-engastada de concreto armado $\left(E=2,88 \times 10^{6} \mathrm{tf} / \mathrm{m}^{2}, \quad V=0.20\right)$ com seção retangular ( $\mathrm{b}=0.15 \mathrm{~m}, \mathrm{~h}=0.60 \mathrm{~m})$, sendo que, o ângulo de abertura é de $60^{\circ}$ e o raio de $8 \mathrm{~m}$, submetida a uma carga uniformemente distribuída de $1.5 \mathrm{tf} / \mathrm{m}$.

Solução

$$
\begin{aligned}
& G=\frac{E}{2 \times(1+v)}=\frac{2.88 \times 10^{6}}{2 \times(1+0.20)}=1.2 \times 10^{6} \mathrm{tf} / \mathrm{m}^{2} \\
& I_{z}=\frac{b x h^{3}}{12}=\frac{0.15 \times(0.6)^{3}}{12}=0.0027 \mathrm{~m}^{4} \\
& x_{t}=\frac{h \times b^{3}}{3}\left[1-0.63 \times\left(\frac{b}{h}\right)+0.052 \times\left(\frac{b}{h}\right)^{5}\right]=0.00056872 \mathrm{~m}^{4} \\
& \text { ExI }=7776 \text { tf } \times \mathrm{m}^{2} \text { (concreto não fissurado) } \\
& \mathrm{GxK}_{t}=682.44 \text { te } \times \mathrm{m}^{2} \text { (concreto nāo figsurado) } \\
& \text { EXI } / 3=2592 \text { tf } \times \mathrm{n}^{2} \text { (concreto fissurado) } \\
& \text { GxK }_{t} / 6=113.74 \text { tf } \times \mathrm{m}^{2} \text { (concreto fiseurado } \\
& \lambda=\frac{E \times I_{z}}{\mathrm{GXK}_{\mathrm{t}}}=22.8 \quad \text { (concreto fissurado) } \\
& \text { Tab. } 5.2 \quad a_{1}=10.513 \quad M_{z}=-10.093 \text { tf } \times \mathrm{m} \\
& \alpha=60^{\circ}+a_{2}=3.329 \rightarrow M_{z}=3.196 \mathrm{tf} \times \mathrm{m} \\
& \lambda=23 \quad a_{3}=0.695 \quad M_{t}=0.667 \mathrm{tf} \times \mathrm{m}
\end{aligned}
$$


MuI. 5 B.

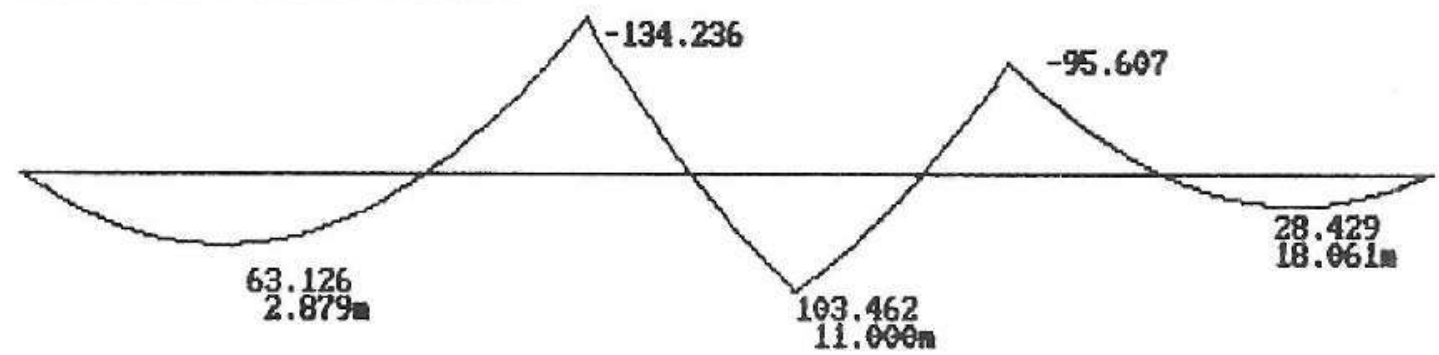

IORCAOS T. UENAN I -KN.

Max $=9.142 \mathrm{kN}$ Pos $=0.0000 \mathrm{~m}$

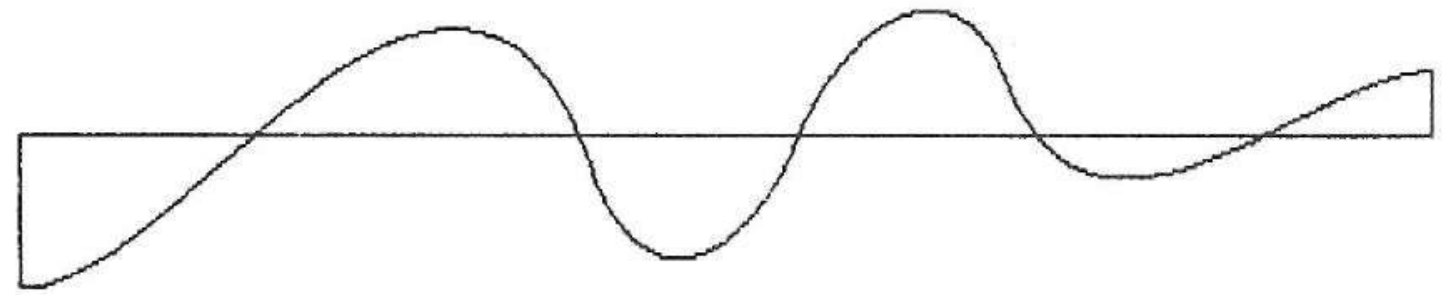

BRCAO EME ENAEN I O- KN.

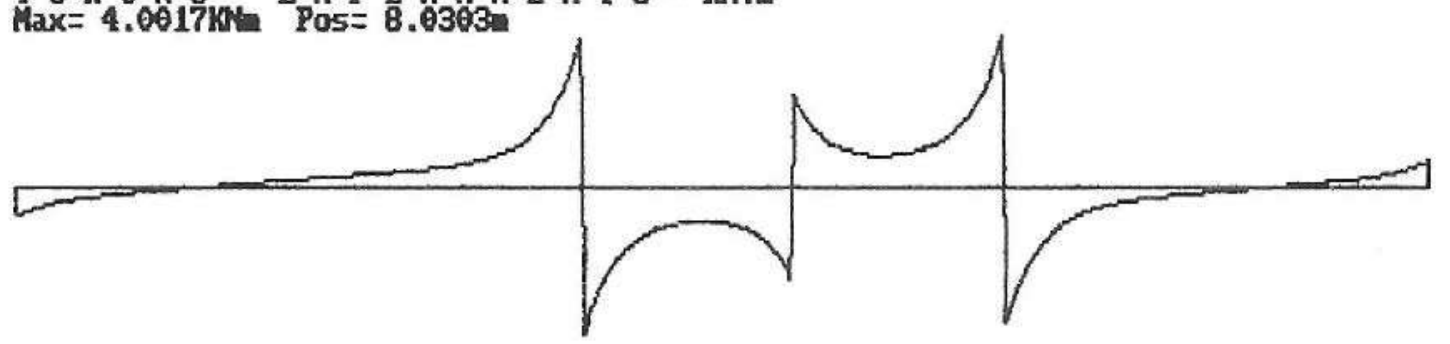

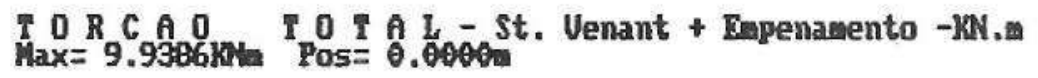

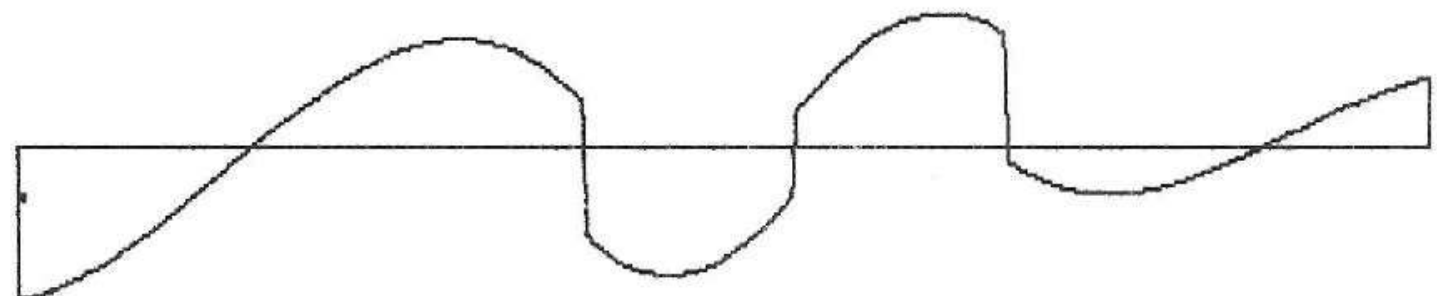

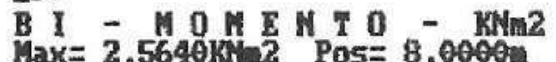

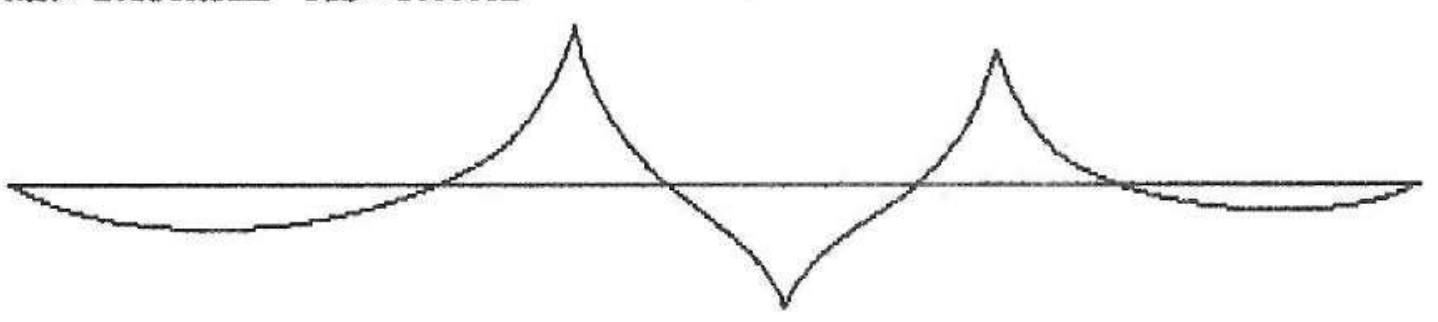

FIGURA 4.5.2 - Diagramas de esforços internos (EXEMPLO 5 - CASO 1) 


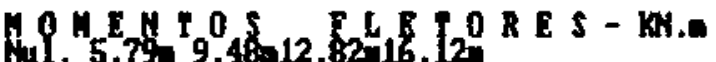

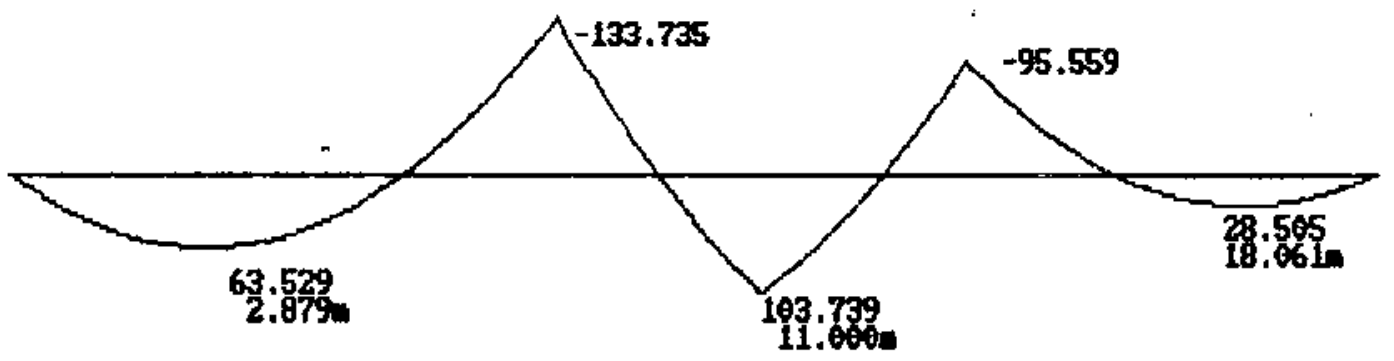

TOR CAO S T. UENANT-ZA.m

Fax= 7.33 bHt Pos=12.9091 -

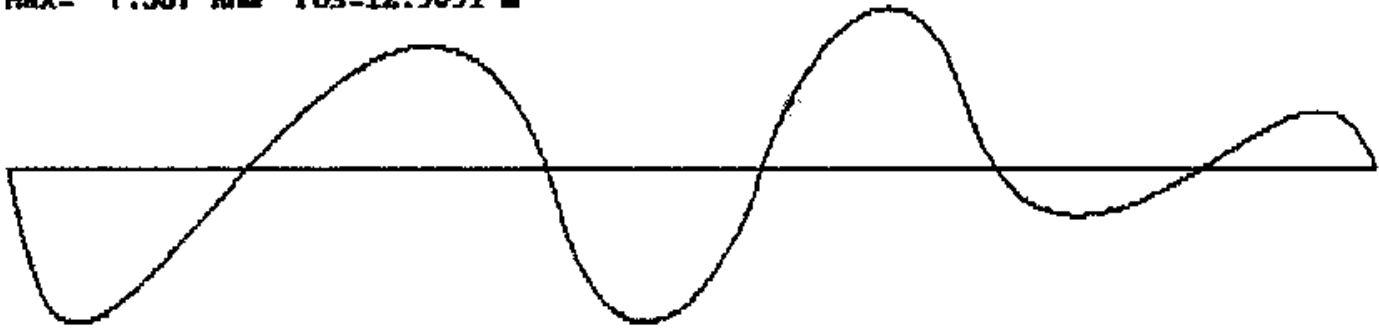

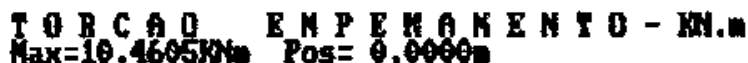

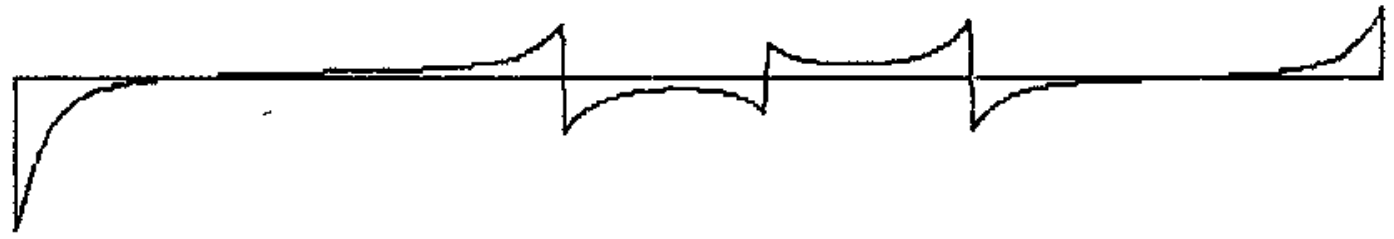

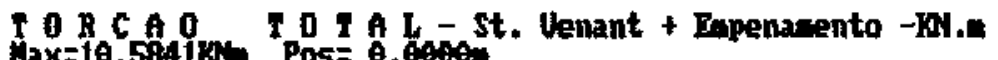
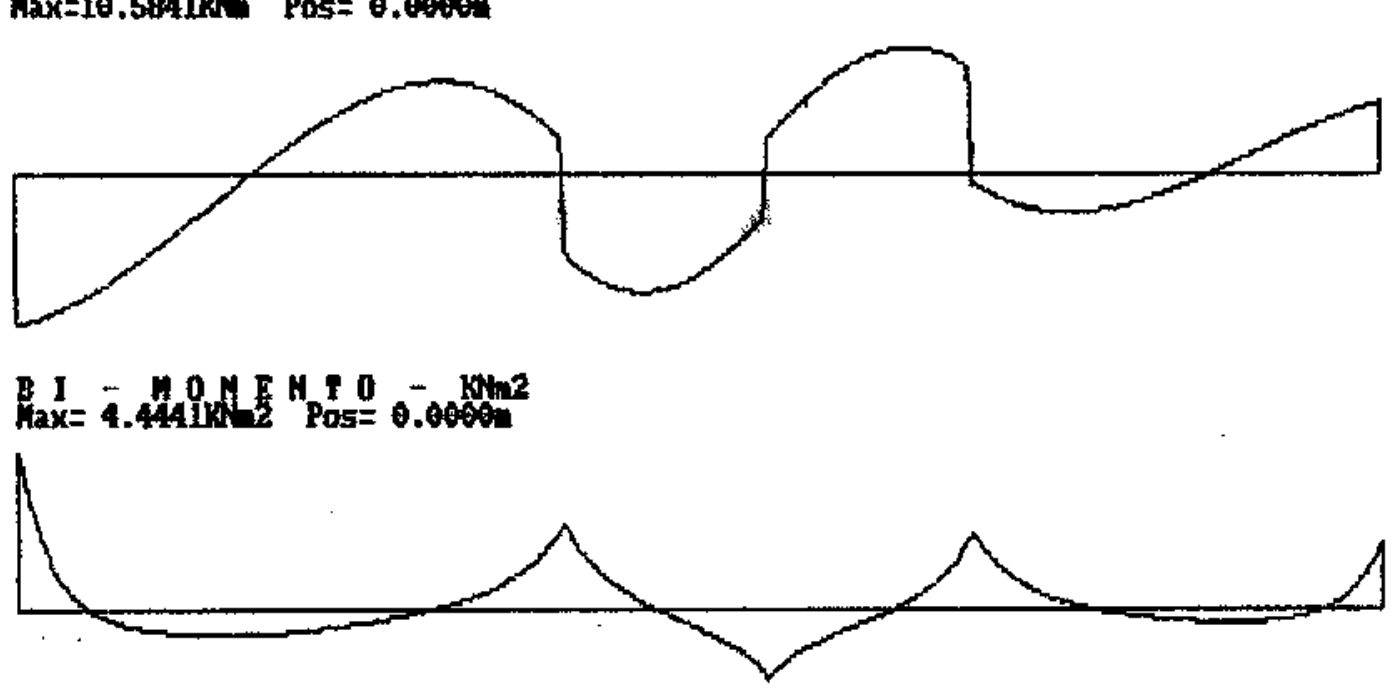

FIGURA 4.5.3 - Diagramas de esforços internos (EXEMPLO 5 - CASO 2) 
$40 \mathrm{MRMTOS}$ TLRTORES- M.

this. 5.910 9.42. $12.68 \mathrm{Bm} 16.66 \mathrm{~m}$

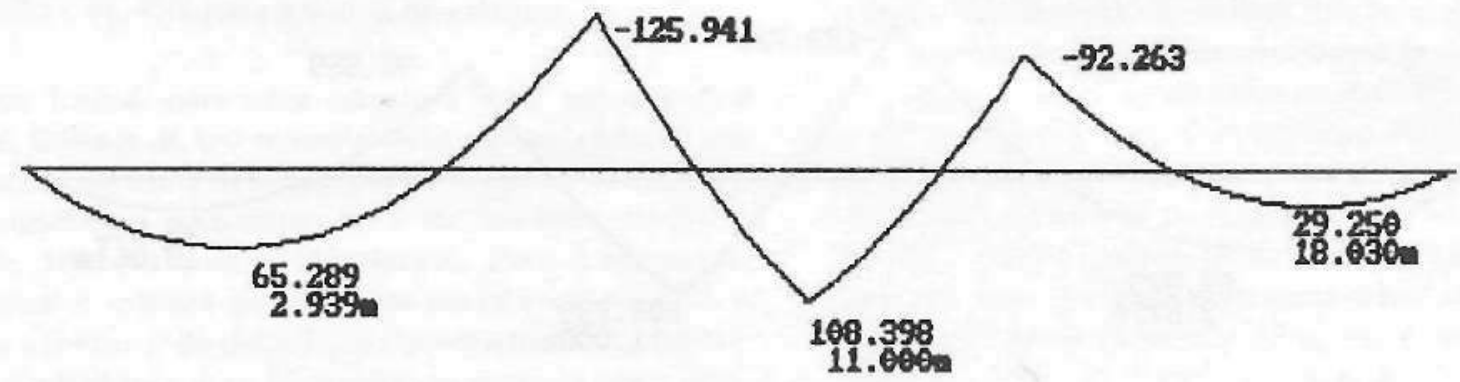

I ORCAO S T. U U NAN T - KN.

Max $=3.952 \mathrm{KNH}$ Pos $=9.6606 \mathrm{~m}$

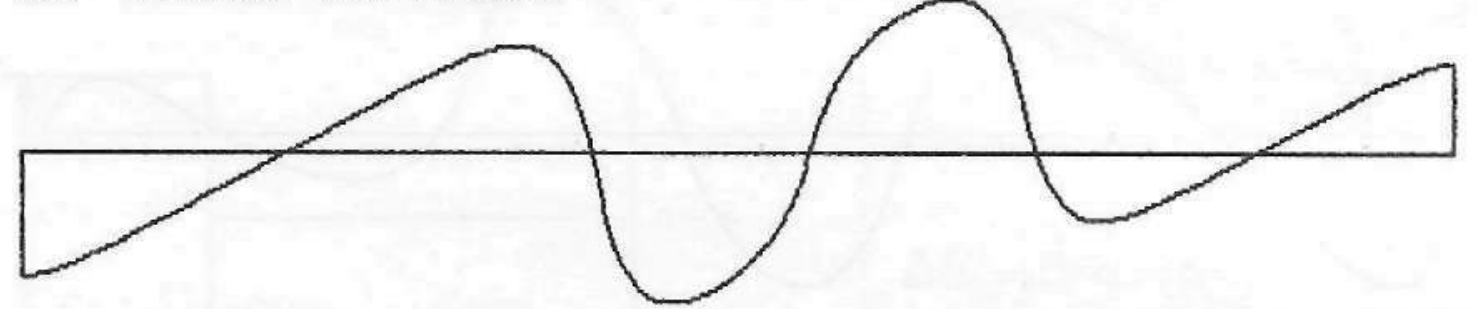

TORCAO E M P E NA

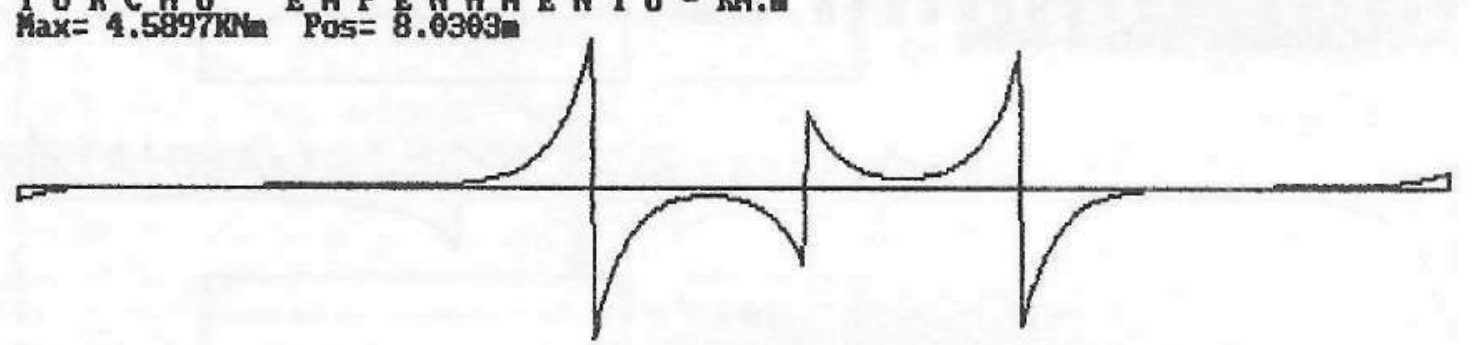

T O R C A O TO A $L-S t$. Venant + Emenamento -KN.

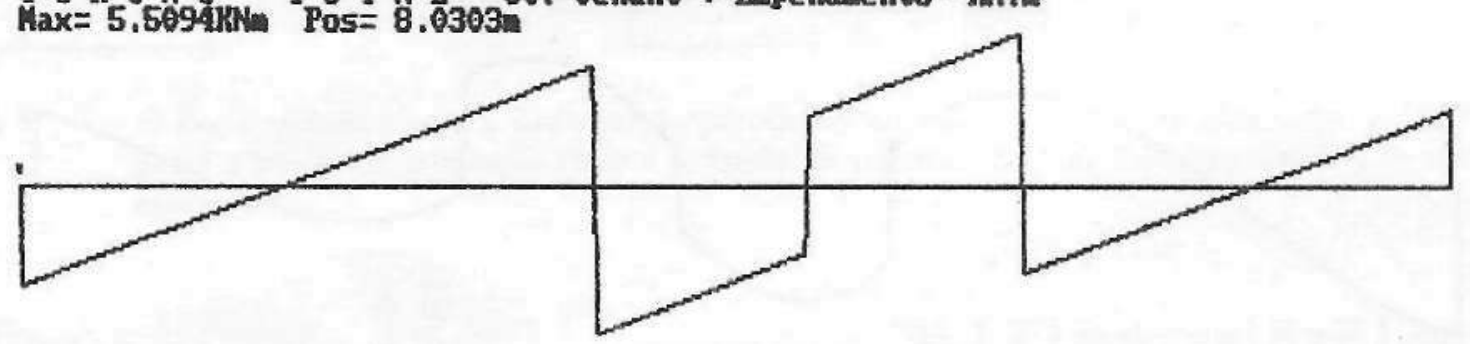

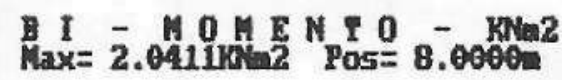

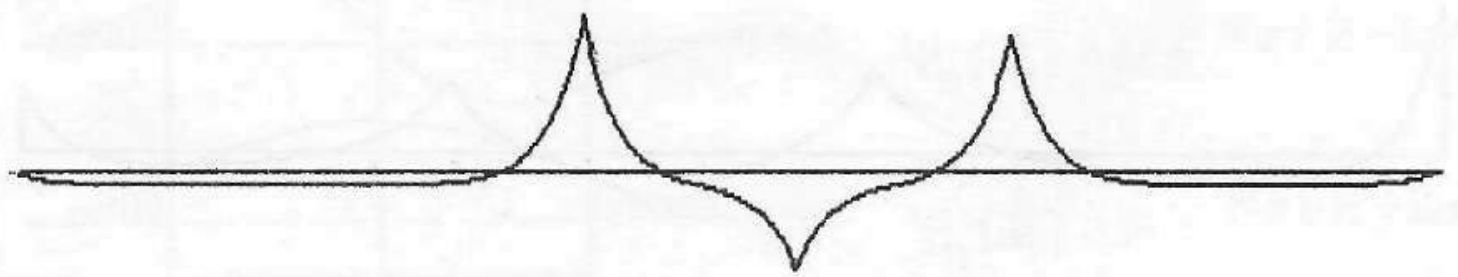

FIGURA 4.5.4 - Diagramas de esforços internos (EXEMPLO 5 - CASO 3) 
TABELA 5.2 - Coeficientes $a_{1}, a_{2}$ e $a_{3}$ funçāo do valor de $\alpha$ e $\bar{\lambda}$.

Engastes: $M_{z}=-a_{1} \mathrm{qR}^{2} / 100 ; \quad$ Meio do väo: $M_{z}=a_{2} \mathrm{qR}^{2} / 100 ; \quad$ Torşor náximo: $M_{t}$ mäx $=a_{3} \mathrm{gR}^{2} / 100$

\begin{tabular}{|c|c|c|c|c|c|c|c|c|c|c|c|c|c|c|c|}
\hline \multirow{2}{*}{\multicolumn{2}{|c|}{$\underset{\text { Graus }}{\alpha}$}} & \multicolumn{14}{|c|}{$\lambda=\mathrm{EI}_{\mathrm{z}} / \mathrm{GK}_{\mathrm{t}}$} \\
\hline & & 0.5 & $\mathbf{1}$ & 2 & 4 & 6 & 8 & 11 & 14 & 17 & 20 & 23 & 26 & 29 & 50 \\
\hline \multirow{3}{*}{15} & $a_{1}$ & 0.57 & 0.57 & 0.57 & 0.58 & 0.58 & 0.58 & 0.58 & 0.58 & 0.58 & 0.58 & 0.59 & 0.59 & 0.59 & 0.60 \\
\hline & $a_{2}$ & 0.29 & 0.29 & 0.28 & 0.28 & 0.28 & 0.28 & 0.28 & 0.28 & 0.28 & 0.27 & 0.27 & 0.27 & 0.27 & 0.26 \\
\hline & $a_{3}$ & 0.01 & 0.01 & 0.01 & 0.01 & 0.01 & 0.01 & 0.01 & 0.01 & 0.01 & 0.01 & 0.01 & 0.01 & 0.01 & 0.01 \\
\hline \multirow{3}{*}{30} & $a_{1}$ & 2.31 & 2.32 & 2.33 & 2.34 & 2.36 & 2.37 & 2.39 & 2.41 & 2.43 & 2.44 & 2.46 & 2.47 & 2.48 & 2.54 \\
\hline & $a_{2}$ & 1.14 & 1.13 & 1.12 & 1.10 & 1.09 & 1.07 & 1.05 & 1.03 & 1.01 & 1.00 & 0.98 & 0.97 & 0.96 & 0.90 \\
\hline & $a_{3}$ & 0.11 & 0.11 & 0.11 & 0.11 & 0.11 & 0.10 & 0.10 & 0.10 & 0.10 & 0.09 & 0.09 & 0.09 & 0.09 & 0.08 \\
\hline \multirow{3}{*}{45} & $a_{1}$ & 5.27 & 5.29 & 5.33 & 5.41 & 5.47 & 5.52 & 5.59 & 5.64 & 5.69 & 5.72 & 5.76 & 5.78 & 5.81 & 5.92 \\
\hline & $a_{2}$ & 2.54 & 2.51 & 2.47 & 2.39 & 2.32 & 2.26 & 2.19 & 2.13 & 2.09 & 2.05 & 2.01 & 1.98 & 1.95 & 1.83 \\
\hline & $a_{3}$ & 0.38 & 0.37 & 0.36 & 0.34 & 0.33 & 0,32 & 0.30 & 0.29 & 0.28 & 0.27 & 0.27 & 0.26 & 0.26 & 0.30 \\
\hline \multirow{3}{*}{60} & ${ }^{a} 1$ & 9.54 & 9.60 & 9,71 & 9.89 & 10.02 & 10.13 & 10.25 & 10.34 & 10.41 & 10.47 & 10.51 & 10.55 & 10.59 & 10.73 \\
\hline & $a_{2}$ & 4.46 & 4.38 & 4.26 & 4.05 & 3.90 & 3.78 & 3.64 & 3.53 & 3.45 & 3.38 & 3.33 & 3.28 & 3.25 & 3.08 \\
\hline & $a_{3}$ & 0.87 & 0.85 & 0.81 & 0.76 & 0.71 & 0.68 & 0.64 & 0.62 & 0.64 & 0.69 & 0.70 & 0.72 & 0.74 & 0.82 \\
\hline \multirow{3}{*}{75} & $a_{1}$ & 15.23 & 15.36 & 15.57 & 15.88 & 16.09 & 16.24 & 16.40 & 16.52 & 16.61 & 16.67 & 16.73 & 16.77 & 16.81 & 16.95 \\
\hline & $a_{2}$ & 6.85 & 6.69 & 6.42 & 6.03 & 5.77 & 5.59 & 5.37 & 5.23 & 5.12 & 5.03 & 4.97 & 4.91 & 4.86 & 4.68 \\
\hline & $a_{3}$ & 1.64 & 1.59 & $1+49$ & 1.36 & 1.27 & 1.21 & 1.30 & 1.39 & 1.50 & 1.51 & 1.56 & 1.59 & 1.61 & 1.73 \\
\hline \multirow{3}{*}{90} & $a_{1}$ & 22.46 & 22.68 & 23.00 & 23.43 & 23.69 & 23.87 & 24.05 & 24.17 & 24.26 & 24.33 & 24.38 & 24.42 & 24.46 & 24.59 \\
\hline & $a_{2}$ & 9.65 & 9.35 & 8.89 & 8.29 & 7.92 & 7.67 & 7.41 & 7.24 & 7.11 & 7.02 & 6.94 & 6.88 & 6.84 & 6.64 \\
\hline & $a_{3}$ & 2.71 & 2.59 & 2.41 & 2.17 & 2.23 & 2.41 & 2.59 & 2.71 & 2.80 & 2.87 & 2.92 & 2.96 & 3.00 & 3.13 \\
\hline \multirow{3}{*}{105} & $a_{1}$ & 31.35 & 31.64 & 32.06 & 32.55 & 32.82 & 33.00 & 33.17 & 33.29 & 33.36 & 33.42 & 33.47 & 33.51 & 33.53 & 33.65 \\
\hline & $a_{2}$ & 12.77 & 12.29 & 11.61 & 10.81 & 10.35 & 10.06 & 9.78 & 9.59 & 9.46 & 9.36 & 9.29 & 9.23 & 9.18 & 9.00 \\
\hline & $a_{3}$ & 4.07 & 3.85 & 3.55 & 3.72 & 4.08 & 4.31 & 4.54 & 4.69 & 4.79 & 4.87 & 4.92 & 4.97 & 5.01 & 5.16 \\
\hline \multirow{3}{*}{120} & $a_{1}$ & 41.97 & 42.30 & 42.74 & 43.21 & 43.46 & 43.61 & 43.75 & & 43.91 & & 43.99 & 44.01 & 44.04 & 44.12 \\
\hline & $a_{2}$ & 16.07 & 15.40 & 14.52 & 13.58 & 13.08 & 12.78 & 12.50 & 12.32 & 12.19 & 12.10 & 12.03 & 11.97 & 11.93 & 11.76 \\
\hline & $a_{3}$ & 5.68 & 5.34 & 5.55 & 6.36 & 6.79 & 7.05 & 7.30 & 7.45 & .7 .56 & 7.64 & 7.70 & 7.75 & 7.79 & 7.93 \\
\hline \multirow{3}{*}{135} & $a_{1}$ & 54.31 & 54.62 & 55.00 & 55.38 & 55.56 & 55.67 & 55.77 & 55.83 & 55.87 & 55.90 & 55.92 & 55.94 & 55.96 & 56.01 \\
\hline & $a_{2}$ & 19.39 & 18.57 & 17.58 & 16.61 & 61.13 & 15.85 & 15.59 & 15.43 & 15.32 & 15.24 & 15.18 & 315.13 & 15.10 & 14.95 \\
\hline & $a_{3}$ & 7.51 & 8.26 & 9.18 & 10.08 & 10.52 & 10.78 & 11.02 & 11.17 & 11.27 & 11.34 & 11.40 & 11.44 & 11.48 & 11.61 \\
\hline \multirow{3}{*}{150} & $a_{1}$ & 68.29 & 68.50 & 68.75 & 68.96 & 69.07 & 69.13 & 69.18 & 69.21 & 69.23 & 69.25 & 69.26 & 669.27 & 69.28 & 69.31 \\
\hline & $a_{2}$ & 22.53 & 21.70 & 20.76 & 19.91 & 19.52 & 19.29 & 19.09 & 18.96 & 18.88 & 18.81 & 18.77 & 18.73 & 18.70 & 18.60 \\
\hline & $a_{3}$ & 12.55 & 13.35 & 14.25 & 15.07 & 15.46 & 15.67 & 15.87 & 15.99 & 16.08 & 16.13 & 16.18 & 16.21 & 16.24 & 16.35 \\
\hline \multirow{3}{*}{165} & $a_{1}$ & 83.65 & 83.73 & 83.81 & 83.88 & 83.91 & 83.93 & 83.95. & 83.96 & 83.96 & 83.97 & 83.97 & 83.97 & 83.98 & 83.98 \\
\hline & $a_{2}$ & 25.26 & 24.66 & 24.02 & 23.49 & 23.25 & 23.11 & 22.99 & 22.92 & 22.87 & 22.84 & 22.81 & 22.79 & 22.77 & 22.71 \\
\hline & $a_{3}$ & 19.80 & 20.40 & 21.03 & 21.56 & 21.80 & 21.93 & 22.05 & 22.12 & 22.17 & 22.20 & 22.23 & 22.25 & $22+27$ & 22.33 \\
\hline \multirow{3}{*}{180} & $a_{1}$ & 99.99 & 99.99 & 99.99 & 99.99 & 99.99 & 99.99 & 99.99 & 99.99 & 99.99 & 99.99 & 99.99 & 99.99 & 99.99 & 99.99 \\
\hline & $a_{2}$ & 27.32 & 27.32 & 27.32 & 27.32 & 27.32 & 27.32 & 27.32 & 27.32 & 27.32 & 27.32 & 27.32 & 27.32 & 27.32 & 27.32 \\
\hline & $a_{3}$ & 29.76 & 29.76 & 29.76 & 29.76 & 29.76 & 29.76 & 29.76 & 29.76 & 29.76 & 29.76 & 29.76 & 29.76 & 29.76 & 29.76 \\
\hline
\end{tabular}

Obs.: Para $\alpha=150^{\circ}$ e $180^{\circ}$ os momentos torçores säo máximos nos engastes, e os demais valores sāo máximos no vāo. Para o concreto armado, deve-se utilizar $\dot{o}$ valor de $\lambda$ levando em con ta o estado fissurado, tanto na flexāo como na torçāo.

Semina, Ci. Exatas/Tecnol., v.12, n.4, p.189-204, dez. 1991 
O método numérico empregado, Diferenças Finitas Centrais, mostrou ser uma ferramenta precisa e confiável, observando uma excelente correlação nos exemplos analisados.

No exemplo 3 e na Tabela 5.1 salienta-se a diferença significativa nos esforços para diferentes relações entre a rigidez a flexão e a torção $\left(E I_{z} / G K_{t}\right)$.

Este trabalho propõe uma tabela que possa ser utllizada por profissionais da área para a obtenção dos esforços internos solicitantes (Momentos Fletores e Torçores). A Tabela 5.1 corresponde a uma viga (ponte) curva bi-engastada a flexão e a torção, quando o ângulo de abertura da viga curva varia de 15 a 180 graus e a relação entre a rigidez a flexão e a torção $\left(E I_{z} / G K_{t}\right)$ varia de 0.5 a 50 .

CARBONARI, G. Static analysis of open thin walled curved continous beams. Semina, v. 12, n. 4, p.189-204, Dec. 1991.

\section{ABSTRACT}

This paper presents a numerical solution to the differential equations resulting from Vlasov Theory of nonsymmetric curved beams with thin walled open cross section. A linear elastic structural behaviour is admitted and the static analysis is performed by using the Method of Central Finite Difference to solve the referred differential equations. Due to the beam curvature, the analysis envolves the coupling between bending and torsion, and both Saint-Venant and warping torsions are taken into account. Beams with any combination of the boundary conditions and intermediary supports are considered, including pinned or fixed ends in torsion and bending. Numerical results obtained through a computer program implemented in a PC - compatible microcomputer system are compared to other technical references, presenting Showed a good correlation.

KEY-WORDS: Curved Beams, Static Analysis, Open Cross, Thin Walled.

\section{REFERÊNCIAS BIBLIOGRÁFICAS}

1 - Carbonari, G. Análise Dinúmica de Vigas Assimétricas em Curva com Parede Fina e Aberta Submetida d̀ Açâo de Cargas Móveis. Tese de Mestrado. Curso de PósGraduaçāo em Engenhạịa Civil, UFRGS, Dezembro, 1986.

2 - VLASOV, V.z. Thin-walled elastic beams. 2. ed. Jerusalem: Israel Program for Scientific Translations, 1961.

3 - TAMAgnA, A. \& HenNemanN, J.C. F. Propriedades Geométricas de seçōes Abertas de Pequena Espessura.
Caderno Técnico CT-36/83, Cursso de Pós-Graduaçāo em Engenharia Civíl - UFRGS, Janeiro, 1983.

4 - HIRSCHFELD, K. Estática en la Construcción. Editorial Reverté, Barcelona, Espanha, 1975.

5 - COURTAND, M. \& LEBELLE, P. Formulaire de Béton Armé. Paxis, 1976. v.2.

Recebido para publicaçāo em 28/4/1992

Semina, Ci. Exatas/Tecnol., v.12, n.4, p.189-204, dez. 1991 